\title{
Momentum, heat and mass transfer simulations of bounded dense mono-dispersed gas-particle systems
}

\author{
Federico Municchi ${ }^{\mathrm{a}, *}$, Stefan Radl ${ }^{\mathrm{a}}$ \\ ${ }^{a}$ Institute of Process and Particle Engineering, TU Graz, Austria
}

\begin{abstract}
Particle Resolved Direct Numerical Simulation (PR-DNS) is employed to study momentum, heat and mass transfer in confined gas-particle suspensions. In this work, we show that the presence of wall boundaries induces an inhomogeneous particle distribution, and as a consequence continuous phase fields exhibit peculiar profiles in the wall-normal direction. Therefore, we first propose a correlation for the particle volume fraction as a function of the distance from the wall and the bulk particle concentration. Secondly, we quantify wall effects on flow field and interphase transfer coefficients (i.e., the flow field, a scalar field, as well as the Nusselt number and drag coefficient). We show that these effects do not depend significantly on the Reynolds number in case an appropriate scaling is applied. Finally, we propose correlations to reconstruct the continuous phase fields in the proximity of adiabatic walls. Also, we provide interpolation tables for the correction to the drag force and the Nusselt number that are helpful in unresolved Euler-Lagrange simulations.
\end{abstract}

Keywords: Particle-resolved direct numerical simulation, closure, wall bounded flows, multiphase flow, heat transfer, gas-solid suspension

\footnotetext{
${ }^{*}$ Corresponding author.
}

E-mail address: fmunicchi@tugraz.at 


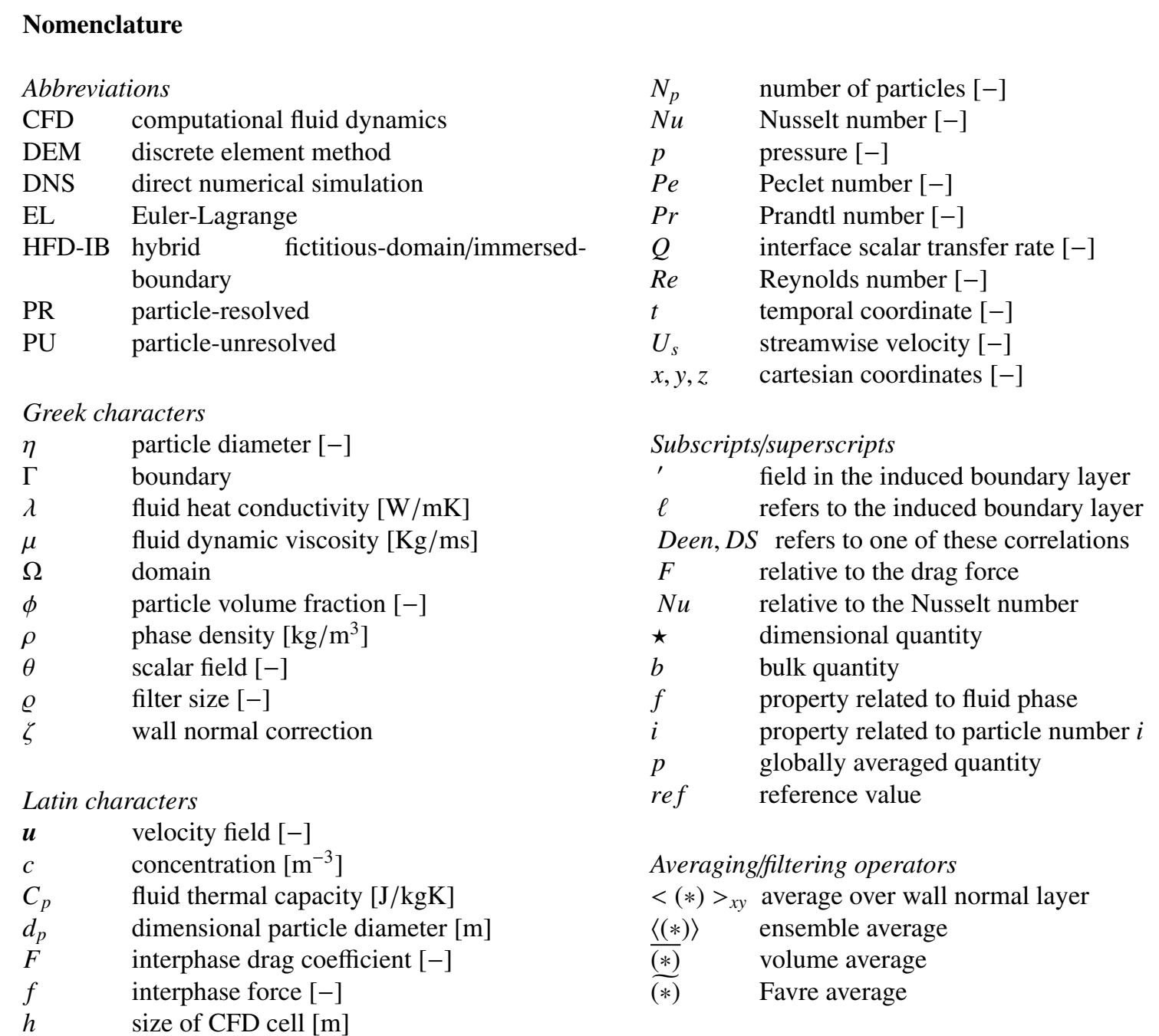

\section{Introduction}

Confined suspensions are a topic of active research since they are of use in a wide range of industrial processes like energy storage, heterogeneous catalytic reactors, pulp fibers, separation in micro-channels, or the petroleum industry. Other applications include blood flow in the human body, sediment transport in river beds and pyroclastic flows from vulcanos. Current developments indicate that the confinement effect in suspension flows becomes even more important: for example, so-called '3D printing' technology aiming on producing materials capable to be used at high temperatures (e.g., metals or ceramics) is already reality [1]. This enables the use of complex geometries with characteristic dimensions closer to that of the suspended particles. In such systems wall effects will play a central role. In addition, the accurate modeling of momentum, heat and mass transport in dense gas-particle systems is of pivotal importance for designing chemical reactors [2, 3], and many other systems, e.g., future solar-thermal systems [4, 5].

Again, the effect of confinement plays a central role in most of these applications, and is potentially becoming more important. However, most of the studies regarding wall effects in fluid-particle systems are devoted to the study of packed beds in cylindrical containments. In contrast, investigations on suspensions bounded by one or more flat walls were performed only recently $[6,7,8,3,9]$. This is despite the obvious importance of near-wall treatment when 
modeling suspension flows: For example, it was shown that (in dilute suspensions under turbulent flow conditions) particles tend to migrate towards (flat) walls due to a phenomenon named turbophoresis [10]. Another example is the peculiar effect that walls have on the particle arrangement in dense suspensions and packed beds as discussed in Section 1.1.

Thanks to the continuous increase in the availability of computational resources, hybrid Computational Fluid Dynamics-Discrete Element Method (CFD-DEM) simulations have become a tool for studying such dispersed multiphase systems [11]. A specific example is the so-called Particle-Unresolved Euler-Lagrange (PU-EL) formulation in which each particle trajectory is followed and particle-particle interactions are resolved. The governing equations for descriping continuous phase flow are formulated at a length scale larger than the particle characteristic length. Therefore, one has to solve coarse-grained equations for the continuous phase. Unlike the Euler-Euler (EE) formulation where the dispersed and continuous phases are described as interpenetrating continua, PU-EL formulations allow to directly study intra-particle transport phenomena. This is possible because PU-EL formulation still retains the definition of single particles as separate discrete entities, allowing to track the internal state of each particle, e.g., intra-particle temperature profiles. Thus, PU-EL simulations are best suited for studying complex systems of chemically reacting particles for which it is difficult (or even impossible) to formulate a continuous dispersed phase model with the desired accuracy. Similarly, modeling systems comprised of non-spherical particles is most natural, and pheraphs successful, when using a EL-based model.

However, coarse grained equations in PU-EL formulations have several unclosed terms (e.g., the drag coefficient, the pseudo-turbulent stress, or the interphase heat and mass transfer coefficients) for which one has to provide suitable expressions. In our previous work [12] we showed how such models can be constructed from Particle-Resolved Euler-Lagrange (PR-EL) simulations by means of volume averaging in a way that is consistent with the PU-EL formulation. It was shown that when the filter size is small (i.e., in the order of two times the particle diameter) significant differences arise with respect to EE closures due to local inhomogeneous structures. In other words, EEbased closures cannot be simply used in PU-EL-based simulation models. Furthermore, PU-EL models perform often poorer compared to EE-based models: inaccuracies caused by the interpolation and mapping scheme used to calculate the local voidage may deteriorate the fidelity of PU-EL models [13]. Particularly interesting aspects surface in case walls are present in the region to be modeled:

- the presence of walls induces an inhomogeneous distribution of particles which affects the flow field and the interphase transfer coefficients. This effect is not acounted for in the totality of closures currently used in EE and PU-EL models.

- since the details of the flow field near the wall are not known, typically the slip condition for the fluid is employed in EE and PU-EL models. This leads to significant uncertainties when interpolating the fluid velocity at the particle position near walls. This issue is especially relevant for size-polydisperse suspensions.

- for PU-EL models, the issue of insufficient mesh resolution in case heterogeneous particle structures exist has been systematically explored only in unbounded domains [14]. One would expect that similar issues arise in case the suspension is confined by walls. Conceptually, one could envision treating such wall effects similar to what is done in wall-bounded turbulent flows (e.g., one could employ wall functions). Unfortunately, such concepts are currently not available for dense fluid-particle flows.

A first step to systematically investigate the above aspects would be to quantify wall effects in an isolated fashion, i.e., separate them from the curvature effect that is typically included in the analysis (see Theuerkauf et al. [15], or van Antwerpen et al. [16]). Also, little is known for more dilute and intermediately dense suspensions, since most previous work explored packed beds only. Considering a wider parameter space is, however, essential when building a robust, generally-applicable simulation model. In our present contribution we indeed show that the particle concentration has a pronounced effect on both the velocity and temperature (as a proxy for any scalar) field. This is even the case for the simplest situation of adiabatic walls. We will start our analysis by considering the origin of these effects, namely the particle distribution near the wall.

\subsection{Particle distribution in wall bounded domains}

Extensive studies have been dedicated to the prediction of particle volume concentration of packed beds in the near wall region. In packed beds, the first layer of spherical particles in contact with the walls is characterized by having 
a well ordered distribution. Most of these near-wall particles are indeed in contact with the wall. Such ordering is progressively lost in the subsequent layers until the particle distribution becomes statistically homogeneous, i.e., the average volume concentration does not vary from one layer to the other anymore.

In earliest works, analytical expressions for the particle volume fraction profile was obtained by volume integration over concentrical annuli in cylindrical packings [17], or over wall-normal layers [18]. More recent studies proposed correlations for these profiles [19, 20], and a comprehensive review of existing correlations is provided by [16]. As discussed above, these studies emphasize on packed beds with cylindrical walls and therefore, they do not distinguish between the effect of the wall curvature and that of the wall alone. Furthermore, most available correlations are based on experimental data. They cover a rather small spectrum of particle volume fractions (typically between 0.35 and 0.65). Surprisingly, there is, to the best of our knowledge, currently no expression for the particle volume fraction as a function of the distance normal to a flat wall for a given particle concentration in the bulk of the particle bed. In our work, the main focus is on suspension flows near flat walls; therefore, we do not aim at improving or replacing existing correlations available for packed beds in cylindrical containers.

\subsection{Momentum, heat and mass transfer in bounded suspensions}

It is not surprising that the peculiar particle ordering near walls leads to a substantial changes in the flow structures: Studies considering cylindrical packed beds revealed that anomalous transport phenomena occur in the fluid that flows in the proximity of a wall boundary [16, 2, 3, 21, 22]. Specifically, it was shown that the inhomogeneous particle distribution leads to characteristic profiles for the flow variables in the direction normal to the wall: For example, the velocity field experiences a parabolic profile (with a characteristic lateral extension of less than one particle diameter) in the vicinity of a wall $[2,23]$. Therefore, considering that the particle volume concentration experiences a local minimum for particles in contact with walls, one may expect that the flow rate in the proximity of walls to be significantly larger than that in the bulk of the bed. This would be especially true in geometries which are characterized by a large particle diameter-to-wall distance ratio (i.e., narrow beds or small cylinders) because the particle volume concentration in the bulk (i.e., center of cylinder or symmetry plane between two walls) will be significantly larger than the average value. Therefore, detailed modeling of near wall perturbations would increase the predictive power of both PU-EL and EE models, since current models cannot account for these effects.

\subsection{Goals and outline}

In the present work, we are studying mometum, heat and mass transfer in dense wall bounded gas-particle suspensions. Specifically, we aim to provide a quantitative description of the wall normal flow and temperature profiles, and we investigate the interphase transfer coefficients in the near-wall region. The ultimate goal is to provide a sound basis for the development of closure models to be used PU-EL simulations that account for the presence of (flat) walls.

The novelties introduced in this work can be summarized in three points, each one representing a topic we attack:

i We quantify the effect of wall boundaries on the particle volume distribution for flat walls. Unlike previous works $[15,16]$, we do not focus on packed beds, but we cover more dilute gas-particle systems for which comparably little is known. The goal is to obtain a sufficiently accurate and computationally affordable correlation to describe wall induced disturbances for a wide range of average particle volume concentrations. Also, we consider flat walls to eliminate curvature effects. This is motivated by (i) the fact that in a typical PU-EL model only the wall-normal distance is known, but not the curvature, and that (ii) the correlation should be as generally-applicable as possible, and hence wall and curvature effect must be separated. This is because simulators of the PU-EL model are coded in such a way that they can deal with arbitrary geometries - typically, no specific information about the walls' shape (e.g., the radius of a cylindrical container) is provided, and a wall is simply represented by a set of triangles.

ii We quantify the effect of such disturbances on the velocity field and a scalar non-reactive field. We put emphasis on quantitative analysis and we provide correlations for all relevant quantities. The ultimate goal of this effort is the replace the primitive slip boundary condition frequently used by a more physical boundary treatment.

iii We investigate the effect of walls on the interphase transfer coefficients (drag coefficent and Nusselt number). This is motivated by the current inability of closures to account for the presence of walls in a generic fashion. 
This work is structured as follows: background on the mathematical description, together with the key quantities is summarized in Section 2. In Section 3 we present details on the numerical solution of the equations presented in Section 2. Results from our simulations are presented in Section 4. In Section 5 we summarize our results and bring them into perspective.

Supplementary material that details the master curves needed to compute the drag and Nusselt numbers as a function of the wall normal distance is available in Appendix A.

\section{Mathematical description}

In the following section we first describe the governing equations to be solved, together with boundary conditions that are imposed in the context of our PR-DNS. Second, we introduce the coarse-graining operators that are employed during the post-processing stage of our study. Such operators allow to smoothen ("filter") the information gained form PR-DNS in order to reproduce the volume-averaged fields that are available (and solved for) in PU-EL formulations.

\subsection{Gas-particle time scale separation and particle velocity fluctuations}

In gas-particle suspensions, the Stokes number (defined as the ratio between the characteristic time scales of particle and fluid flow) is generally very high due to the large density ratio between the two phases. This can be understood by considering an external force (e.g., due to an imposed pressure gradient) acting on equal volumes of the fluid and solid phase: since the gas and particle densities are significantly different, so is their inertia. Therefore, one of the two phases is responding much faster than the other to the presence of the external force. In the limit of an infinitely high Stokes number, and when considering interphase transfer (e.g., drag forces or heat flux) the gas phase is going to relax towards an attractor (for example a steady state if it exists) much faster than particles do. Thus, gas flow will not be influenced by a change in the state of the particle phase (e.g., particle position, velocity or temperature). Furthermore, since in gas-particle systems the Prandtl number is close to unity, the characteristics length and time scales of velocity and temperature field dynamics will be very similar.

Therefore, we may argue that the time scales for the evolution of particle and fluid phase properties are much different, and that we can study the combined dynamics as a sequence of fixed particle beds, each one with a temporally developed fluid flow.

Following the previous considerations, we consider a static isothermal particle bed, i.e., particles are not allowed to move or to change their temperature. For example, Holloway et al.[24] employed the very same approach (that they named 'frozen particle approach') in a previous study that focused on the prediction of fluid-particle drag forces.

While particles in a gas-particle systems were often considered as 'frozen', this representation neglects the particles' fluctuating speed around the mean particle speed. Such fluctuations have been recently shown to play a significant role in the correct evaluation of the drag coefficient [25]. Specifically, the effect of particle velocity fluctuations can be accounted for by introducing an additional term in the drag expression that depends on the "particle temperature Reynolds number" (defined using the square root of the fluctuating kinetic energy of the particle cloud). In our work we will consider particle-based interphase transfer coefficients, i.e., quantities that take the effect of individual fluidparticle relative speeds into account. Hence, our approach incorporates aready some (but not all) of the effects studied by Tang et al. [25]. What we will leave out in our present study is the effect that the speed of neighbouring particles has on these particle-individual transfer coefficients. This is done on purpose, since no expression for the particleindividual interphase heat and mass transfer coefficients (far away from walls) exists in literature that accounts for "particle temperature Reynolds number" effects.

Therefore, in our study we neglect particle velocity fluctuations, and we assume that particles are arrested (i.e., they do not move relative to the wall). Hence, our study considers gas-particle suspensions where the average fluid-particle relative speed is much larger than particle velocity fluctuations, and in which the wall has the particle-average speed. We note in passing that an extension to systems having non-zero wall-particle relative speeds is straight forward, but not in the focus of our present study.

\subsection{Transport equations}

In the present work, we solve the incompressible Navier-Stokes equations together with an advection-diffusion equation for a dimensionless inert scalar $\theta(t, \boldsymbol{x})$ to model momentum, heat and mass transport in dense gas-particle 
systems. The governing equations are formulated in their dimensionless form, and are defined within the fluid domain $\Omega_{f}$ :

$$
\begin{aligned}
& \boldsymbol{\nabla} \cdot \boldsymbol{u}(t, \boldsymbol{x})=0 \\
& {\left[\partial_{t}+\boldsymbol{u}(t, \boldsymbol{x}) \cdot \boldsymbol{\nabla}-R e^{-1} \nabla^{2}\right] \boldsymbol{u}(t, \boldsymbol{x})=-\boldsymbol{\nabla} p(t, \boldsymbol{x})} \\
& {\left[\partial_{t}+\boldsymbol{u}(t, \boldsymbol{x}) \cdot \boldsymbol{\nabla}-P e^{-1} \nabla^{2}\right] \theta(t, \boldsymbol{x})=0}
\end{aligned}
$$

Where $\boldsymbol{u}$ is the dimensionless velocity field, $p$ is the dimensionless (dynamic) pressure, Re is the Reynolds number, $\theta$ is the dimensionless inert scalar field, and $P e$ is the Peclet number defined as $P e=\operatorname{RePr}$ being $\operatorname{Pr}$ the Prandtl number. In this work, we will always set $P r=1$ so that $P e=R e$ and therefore, the differential operators on the left hand side of $1 \mathrm{~b}$ and 2 are the same. Equations 1 and 2 are valid for $\boldsymbol{x} \in \Omega_{f}$

\subsubsection{Dimensional flow variables}

In order to avoid confusion in the scaling process of equations 1 and 2 we briefly introduce the procedure we used for obtaining dimensionless quantities:

i All length scales are made dimensionless with the particle diameter $d_{p}$.

ii The dimensionless velocity field $\boldsymbol{u}$ is obtained by scaling the dimensional velocity field with a reference velocity magnitude $U_{r e f}$. The latter is determined from the Reynolds number using $U_{r e f}=(\operatorname{Re} \mu) /\left(\rho d_{p}\right)$ being $\mu$ the dynamic fluid viscosity and $\rho$ the fluid density.

iii Time scales were made dimensionless employing (i) and (ii). Therefore, the dimensional time scales with $d_{p} / U_{\text {ref }}$.

iv The dimensional (dynamic) pressure scales with $\rho U_{r e f}^{2}$. Similarly, a component of the dimensionless stress tensor $\tau_{x z}=R e^{-1} \partial_{z} u_{x}$ (with $\boldsymbol{u}=\left(u_{x}, u_{y}, u_{z}\right)$ ) is related to its dimensional value by a multiplicative factor of $\rho U_{\text {ref }}^{2}$.

$\mathrm{v}$ Since we consider a constant surface scalar value $c_{s}$, which is the same for each particle, we defined the dimensional scalar as $c=\theta\left(c_{s}-c_{i}\right)+c_{i}$ where $c_{i}$ is a reference value of the scalar whose meaning is give from Eqn. 6 shown below. We note in passing that such a scaling is possible due to the linearity of equation 2 .

Following the above procedure, and considering that $P e=\left(\rho C_{p} U_{r e f} d_{p}\right) / \lambda_{f}$ (where $\lambda_{f}$ is the fluid heat conductivity and $C_{p}$ is the fluid's specific heat capacity), we can conclude that the interphase heat source for particle $i$ scales as $d_{p}^{-1} \rho C_{p} U_{r e f}\left(c_{s}-c_{i}\right) Q_{i}$. Here $Q_{i}$ is the dimensionless interphase scalar transfer rate of particle $i$. Otherwise, if one applies equation 2 to the transport of some inert substance in the fluid, then our Peclet number $P e$ can be expressed as $P e=\left(U_{r e f} d_{p}\right) / \mathscr{D}$, where $\mathscr{D}$ is the diffusion coefficient. The interphase source term is then defined via $d_{p}^{-1} U_{r e f}\left(c_{s}-c_{i}\right) Q_{i}$.

Similarly, the interphase force $f_{i}$ was made dimensionless using a scaling factor of $\rho U_{s}^{2} / d_{p}$.

\subsection{Boundary conditions}

We define a global domain $\Omega=\Omega_{f} \cup \Omega_{i}=\left[0, L_{x}\right] \times\left[0, L_{y}\right] \times\left[0, L_{z}\right]$, where $\Omega_{i}$ is the region occupied by particle $i$ and where $L_{x}, L_{y}$ and $L_{z}$ represent the extension of the global domain in the $x, y$ and $z$ directions, respectively. We can define two kind of boundaries: fluid-particle boundaries $\Gamma_{i}$ and a global domain boundary $\Gamma_{g}$. Standard Neumann boundary conditions are employed for the pressure field at walls. At particle surfaces we apply the following boundary conditions:

$$
\boldsymbol{u}(t, \boldsymbol{x})=\mathbf{0} \quad \text { and } \quad \theta(t, \boldsymbol{x})=\theta_{s}, \quad \forall \boldsymbol{x} \in \Gamma_{i}, \quad i=1,2, \ldots, \mathrm{N}_{p}
$$


Where $\mathrm{N}_{p}$ is the number of particles in $\Omega$ and $\theta_{s}$ is the particle surface scalar value. In the present study, $\Gamma_{g}$ is a semi-periodic boundary with adiabatic walls where the following boundary conditions apply:

$$
\begin{aligned}
& \boldsymbol{u}(t, 0, y, z)=\boldsymbol{u}\left(t, L_{x}, y, z\right), \quad \theta(t, 0, y, z)=\theta\left(t, L_{x}, y, z\right) \\
& \boldsymbol{u}(t, x, 0, z)=\boldsymbol{u}\left(t, x, L_{y}, z\right), \quad \theta(t, x, 0, z)=\theta\left(t, x, L_{y}, z\right) \\
& \boldsymbol{u}(t, x, y, 0)=\boldsymbol{u}\left(t, x, y, L_{z}\right)=\mathbf{0},\left.\quad \partial_{z} \theta\right|_{z=0}=\left.\partial_{z} \theta\right|_{z=L_{z}}=0
\end{aligned}
$$

Naturally, such problem would approach the trivial solution $\boldsymbol{u}=\mathbf{0}$ and $\theta=1$ everywhere in $\Omega_{f}$ within a time that depends on the boundary and initial conditions. In order to obtain a meaningful solution, one has to (i) impose a mean flow, i.e. a pressure gradient and (ii) prevent saturation phenomena in the fluid phase [12, 26]. We therefore impose the following normalization condition for the dimensionless velocity field in the direction $\boldsymbol{n}_{x}=(1,0,0)$ :

$$
f_{\Omega_{f}} \boldsymbol{u} \cdot \boldsymbol{n}_{x} \mathrm{~d} x \mathrm{~d} y \mathrm{~d} z=f_{\Omega_{f}} U_{s} \mathrm{~d} x \mathrm{~d} y \mathrm{~d} z=1, \quad \forall t \in \mathbb{R}^{+}
$$

Where the operator $f$ is a shorthand notation for the integral mean in the integration domain, i.e., the volume integral of a quantity normalized with the volume. Constraint 5 can be considered as a mean flow condition and the scalar $U_{s}=\boldsymbol{u} \cdot \boldsymbol{n}_{x}$ indicates the flow in the streamwise direction.

In order to prevent saturation phenomena, we adopt the same strategy we used in our previous work [12]: a heat sink is positioned at $0<x<\epsilon$ where $\epsilon=d_{p} / 10$ and $d_{p}$ is the particle diameter. This is equivalent to imposing the following condition on $\Omega_{\text {sink }}=\Omega_{f} \cap[0, \epsilon] \times\left[0, L_{y}\right] \times\left[0, L_{z}\right]$ :

$$
f_{\Omega_{\text {sink }}} \theta \mathrm{d} x \mathrm{~d} y \mathrm{~d} z=0, \quad \forall t \in \mathbb{R}^{+}
$$

\subsection{Volume averaging operator}

In the field of particulate flows and porous media, volume averaging is often employed to derive coarse grained equations that describe the system in terms of integral mean values of the original fields [27]. This approach is also known as the representative volume method [28] whenever the governing equations are filtered within a sufficiently large volume and homogenized diffusion coefficients are then obtained.

In PU-EL formulations, the volume of averaging corresponds to the Eulerian grid cell which is generally a hexahedron. Therefore, we define the averaging volume as $\Omega_{\varrho}(\boldsymbol{x})=\left\{\boldsymbol{x}^{\prime} \in \Omega \quad \mid \quad(\boldsymbol{x}-\varrho / 2) \leq \boldsymbol{x}^{\prime} \leq(\boldsymbol{x}+\varrho / 2)\right\}$, where the inequality is valid for each vector component and where $\varrho$ is the dimensionless filter size. In the following, we will consider cubic averaging volumes and therefore, we will use $\varrho$ to indicate a generic component of $\varrho$. Let's take the scalar field $\theta(t, \boldsymbol{x})$ (the extension to the other fluid fields is trivial). We define the respective continuous phase filtered (i.e., volume averaged) field $\widetilde{\theta}(t, \boldsymbol{x})$ as:

$$
\widetilde{\theta}(t, \boldsymbol{x})=\int_{\Omega_{f} \cap \Omega_{\varrho}(\boldsymbol{x})} \theta\left(t, \boldsymbol{x}^{\prime}\right) \mathrm{d} x^{\prime} \mathrm{d} y^{\prime} \mathrm{d} z^{\prime}
$$

Definition 7 is however not of practical use since (i) $\Omega_{f}$ is generally a function of time, and since (ii) the information regarding the discrete phase (e.g., the particle configuration) remains implicit inside the integral. Therefore, it is general practice to extend the continuous phase fields to the whole $\Omega$ and to define the following indicator function:

$$
\phi(t, x)= \begin{cases}0 & x \in \Omega_{f} \\ 1 & \text { otherwise }\end{cases}
$$


One advantage of PU-EL formulations with respect to EE formulations, is that the indicator function is known from the solution of the Newton's equation of translational motion for the particle cloud. Therefore, we define a mean particle volume fraction:

$$
\bar{\phi}(t, \boldsymbol{x})=f_{\Omega_{Q}(x)} \phi\left(t, \boldsymbol{x}^{\prime}\right) \mathrm{d} x^{\prime} \mathrm{d} y^{\prime} \mathrm{d} z^{\prime}
$$

which can be easily calculated from PU-EL results since particle positions and diameters (which are required to define $\phi$ ) are known.

Finally, we define the voidage $\phi_{f}(t, \boldsymbol{x})=1-\phi(t, \boldsymbol{x})$ and the mean voidage $\overline{\phi_{f}}(t, \boldsymbol{x})=1-\bar{\phi}(t, \boldsymbol{x})$ to obtain:

$$
f_{\Omega_{f} \cap \Omega_{\varrho}} \theta\left(t, \boldsymbol{x}^{\prime}\right) \mathrm{d} x^{\prime} \mathrm{d} y^{\prime} \mathrm{d} z^{\prime}=\frac{1}{\overline{\phi_{f}}(t, \boldsymbol{x})} f_{\Omega_{\varrho}(\boldsymbol{x})} \phi_{f}\left(t, \boldsymbol{x}^{\prime}\right) \theta\left(t, \boldsymbol{x}^{\prime}\right) \mathrm{d} x^{\prime} \mathrm{d} y^{\prime} \mathrm{d} z^{\prime}=\frac{\overline{\phi_{f} \theta}}{\overline{\phi_{f}}}=\widetilde{\theta}(t, \boldsymbol{x})
$$

This operation is equivalent to the Favre averaging operator often used in compressible turbulence. However, it should be noticed that our definition of Favre averaging has nothing to do with compressibility but instead, arises due to the presence of a dispersed phase.

Furthermore, in our notation we deliberately omitted the dependence on $\varrho$ of the volume averaged fields and in the following we will refer to volume averaged fields for different values of the dimensionless filter size.

Another kind of averaging we employ is the wall normal layer averaging. This is defined, for the particle volume concentration $\phi$, as:

$$
<\phi>_{x y}(z)=f_{\Omega_{x y}} \phi(x, y, z) \mathrm{d} x \mathrm{~d} y
$$

While, for the other flow variables (e.g., $\theta)$ :

$$
<\theta>_{x y}(z)=\frac{1}{<\phi_{f}>_{x y}(z)} f_{\Omega_{x y}} \phi_{f}(x, y, z) \theta(t, x, y, z) \mathrm{d} x \mathrm{~d} y
$$

In the following, we will also define bulk quantities (indicated by subscript $b$ ) which satisfy (e.g., in the case of $\theta$ ):

$$
\theta_{b}=\int_{z_{\ell}}^{L_{z} / 2}<\theta>_{x y}(z) \mathrm{d} z \quad \text { where } \quad z_{\ell}: \theta_{b} \gg\left[<\theta>_{x y}(z)-\theta_{b}\right], \quad \forall z \in\left[z_{\ell}, L_{z} / 2\right]
$$

Therefore, bulk quantities are defined in a region located at a larger distance than $z_{\ell}$ from the wall. In this region the maximum deviation of the wall normal layer averaged field from a global mean value is negligible. Such a global mean value is what we call the bulk value. In other words, wall effects in the bulk region can only be of zero order as we describe in Section 4.1.

Finally, we define the overall mean particle volume concentration $\phi_{p}$ as:

$$
\phi_{p}=f_{\Omega} \phi\left(x^{\prime}, y^{\prime}, z^{\prime}\right) \mathrm{d} x^{\prime} \mathrm{d} y^{\prime} \mathrm{d} z^{\prime}
$$

A graphical interpretation of the filtering operators is given in Figure 1. 


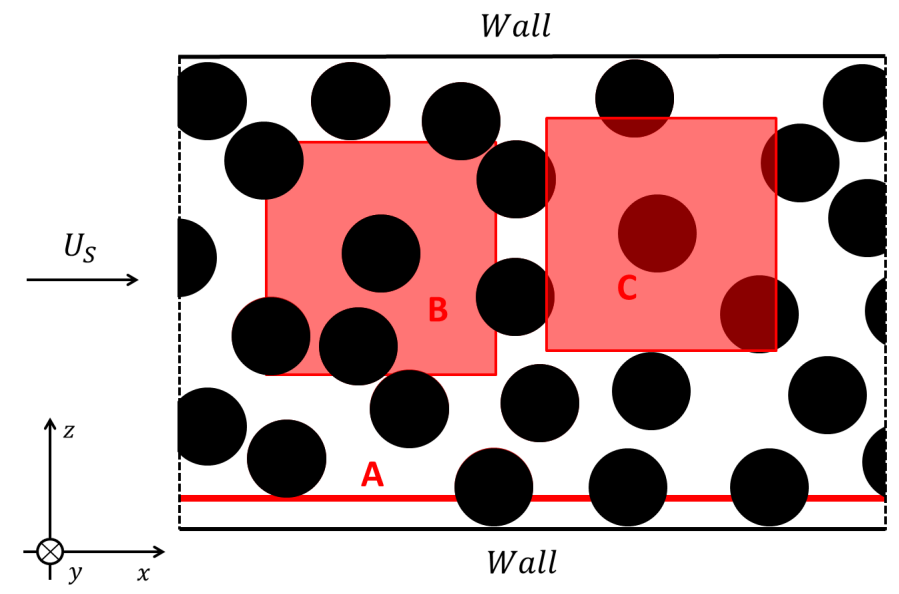

Figure 1: Integration domains relative to different filtering operators. Line A represents the integration domain corresponding to the operator $<(*)>_{x y}$; such domain is just a function of the wall normal coordinate $z$. Region B corresponds to the integration domain of operator $(*)$, therefore the fluid domain bounded by the filter limits (red lines). Region $\mathrm{C}$ corresponds to the operator $\overline{(*)}$, and represent the total volume within the filter limits (red lines).

\subsection{Particle-based interphase transfer coefficients}

In PU-EL simulations, closures for interphase transfer coefficients must be provide at a particle level, i.e., each particle exchanges a different quantity of momentum (drag force) and scalar (scalar transfer). Averaged fields experienced by each particle $i$ are computed by interpolating neighbouring cell values at the particle location $\boldsymbol{x}_{i}$. To account for such interpolation process (typically, a linear interpolation is employed), we will always consider averaging volumes centred at the particle location, i.e., $\Omega_{\varrho}\left(\boldsymbol{x}_{i}\right)$.

Generally, the (dimensionless) drag coefficient $F_{i}$ characterizing fluid-particle momentum exchange associated with particle $i$ is obtained by scaling the drag force with a Stokes-like reference drag force. In case a dimensionless drag force $f_{i}$ is already available (see Section 2.2.1), $F_{i}$ can be computed from a dimensionless Stokes-like reference drag force via:

$$
F_{i}=\frac{\boldsymbol{f}_{i} \cdot \boldsymbol{n}_{x} R e}{3 \pi \eta_{i}\left[1-\bar{\phi}\left(t, \boldsymbol{x}_{i}\right)\right] \widetilde{\boldsymbol{u}}\left(t, \boldsymbol{x}_{i}\right) \cdot \boldsymbol{n}_{x}}
$$

Where $\eta_{i}$ is the dimensionless particle diameter, which is unity for all particles in case of monodisperse systems as considered in our present study. We note in passing that 'drag' refers in our study only to force components in the main flow direction $\boldsymbol{n}_{x}$. Certainly, flow induced fluid-particle interaction forces arise also in other direction. However, our preliminary work showed that these force components are of subordinate importance on average, and hence are not discussed in greater detail in what follows.

The interphase scalar source term $Q_{i}$ is generally accounted for via the particle-based Nusselt (or Sherwood) number defined as:

$$
N u_{i}=\frac{Q_{i} P e}{\pi \eta_{i}\left[\theta_{s}-\widetilde{\theta}\left(t, \boldsymbol{x}_{i}\right)\right]}
$$




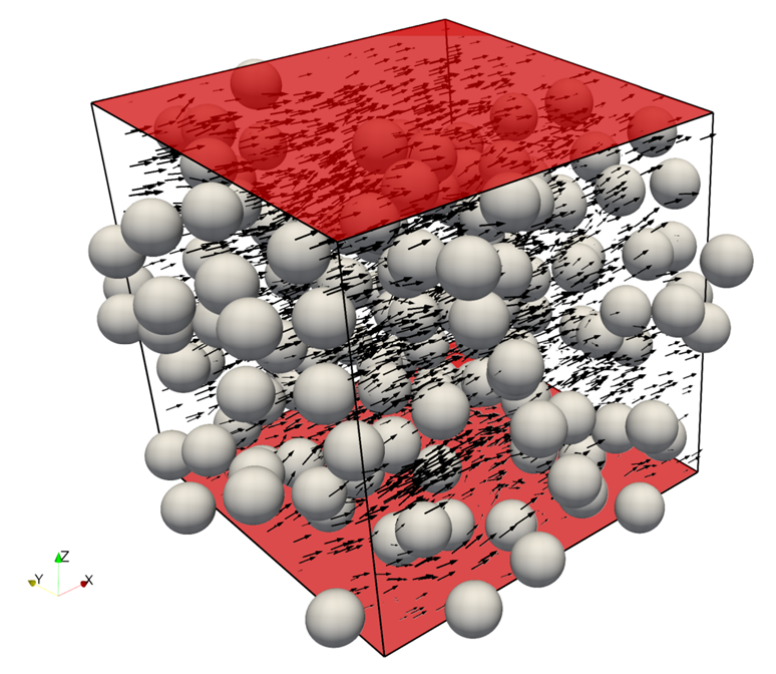

Figure 2: Computational domain $\left(\phi_{p}=0.1, R e=100\right)$. Red surfaces correspond to wall boundaries and arrows represent the directions of the instantaneous velocity field.

\section{Numerical formulation}

In the present work, we used the finite volume library OpenFOAM ${ }^{\circledR}[29]$ together with Euler-Lagrange library CFDEMCoupling ${ }^{\circledR}[30]$ to solve the governing equations. We ran simulations for several values of $\phi_{p}$ and $R e$ as shown in Table 1. The number of realizations was such that we considered always more than 1,000 individual particles for each combination of $\phi_{p}$ and $R e$. This is necessary to collect meaningful statistics as a function of the wall-normal distance.

Table 1: Cases analysed in the current study.

\begin{tabular}{ccc}
\hline$\phi_{p}$ & $R e$ & Number of realizations \\
\hline 0.1 & $100,200,300,400$ & 10 \\
0.2 & $100,200,300,400$ & 5 \\
0.3 & $100,200,300,400$ & 4 \\
0.4 & $100,200,300,400$ & 3 \\
\hline
\end{tabular}

\subsection{Bed generation}

Each particle configuration was generated running a granular-only DEM-based simulation using LIGGGHTS ${ }^{\circledR}[31]$. Specifically, the desired global-mean particle volume fraction was realized by shrinking a box containing initially randomly distributed particles. Particles were let free to collide (with other particles, as well as the the wall boundaries in the z-direction) until the final particle configuration was reached. The final size of the shrunken bounding box was set to $8 d_{p} \times 8 d_{p} \times 8 d_{p}$. The so obtained configurations were subsequently used as the input for the CFD simulation, in which particles were arrested with zero speed.

The so obtained configurations were subsequently used as the input for the CFD simulation, in which particles were arrested with zero speed. Figure 2 shows the computational domain corresponding to one of the configurations investigated. 


\subsection{Mesh generation and CFD solution}

We used the OpenFOAM ${ }^{\circledR}$ library together with CFDEMCoupling ${ }^{\circledR}$ to assemble a segregated algorithm that solves the governing equations as described in our previous work [12] where we proposed the Hybrid Fictitious DomainImmersed Boundary method (HFD-IB). The use of an immersed boundary approach allows to solve the governing equations in a global domain $\Omega$ with simple shape (which can be efficiently meshed using a simple Cartesian grid) rather than the highly complex fluid domain $\Omega_{f}$. This is why immersed boundary methods are frequently used in the field of suspension flows, even in case particles are arrested (see, for example in [8] or [32, 33, 34, 35, 36, 37]). The HFD-IB approach ensures that no errors arise due to highly skewed cells and, most important, it eliminates the effort to build a body-fitted mesh. Furthermore, using the IB method facilitates the decomposition of volume average integrals presented in Section 2.4. A drawback of IB method-based approaches is that an accurate representation of the immersed surfaces requires a large number of interpolation points, or a mesh refinement in the proximity of the surfaces [12]. This is since the addition of layers consisting of "thin" cells aligned with the immersed surfaces is not possible when using IB methods. To alleviate this drawback, careful grid sensitivity studies are necessary when using IB methods. Specifically, we found that a grid of size $h=d_{p} / 20$ did not produce significantly different results from a grid of size $h=d_{p} / 50$ as we used in our previous work [12]. This can be attributed to the scaling technique we employed in our statistical analysis, which removes the dependency on the Reynolds number, and therefore alleviates the necessity of an extremely fine grid. To support this with data, we performed a verification study (see Section 4.3). Specifically, we show that the bulk value of the particle based Nusselt number agrees favourable with correlations available in literature.

We adopted a second order accurate discretization in space for all terms, and a backward time integration scheme. A diagonal incomplete-Cholesky preconditioned conjugate gradient method was employed to solve the Poisson equation for the pressure field. The momentum and scalar transport equations were solved using a diagonal incomplete lower-upper preconditioned bi-conjugate gradient method. Conjugate gradient methods were preferred to multigrid methods due to the presence of singular forcing terms in our discretised equations.

Convergence and algorithmic details are identical to our previous work [12].

\subsection{Post processing}

We employed the open source library CPPPO [38] to perform all post processing steps. Specifically, this consisted of (i) volume averaging over wall normal layers, or (ii) over $\Omega_{\varrho}\left(\boldsymbol{x}_{i}\right)$ for each particle. As described in our previous work on bi-dispersed suspensions [12], averaging the scalar field over periodic boundaries poses a problem due to the field being statistically inhomogeneous in the streamwise direction. Therefore, only particles whose related volume averaging domains $\Omega_{\varrho}\left(\boldsymbol{x}_{i}\right)$ do not cross the periodic boundaries normal to the streamwise direction are considered when probing the quantities related to scalar transport.

\section{Results}

After applying the numerical schemes summarized in Section 3, as well as the volume averaging operations from Section 2.4, a large set of raw data was collected. This data included per-particle, as well as layer-averaged flow quantities for multiple realizations of each flow situation. With flow situation we refer to a specific arrangement of particles, as well as the steady-state flow profile that is characterized by the Reynolds number and the domain-average particle volume fraction. The following results have been obtained after ensemble averaging over the respective subset of the raw data.

\subsection{Wall-normal particle distribution}

Figure 3 shows the wall normal profile of the particle volume fraction for different values of $\phi_{p}$. In current literature, these profiles are often called wall radial distribution function due to the cylindrical geometries employed in their evaluation. In what follows it is useful to consider these profiles as perturbations with respect to the homogeneous (bulk) region. We can clearly see that both (i) the absolute amplitude, and (ii) the wave number of the perturbation are larger for larger values of $\phi_{p}$. This can be related to the higher degree of order that the particles experience near the wall at higher packing fractions. 


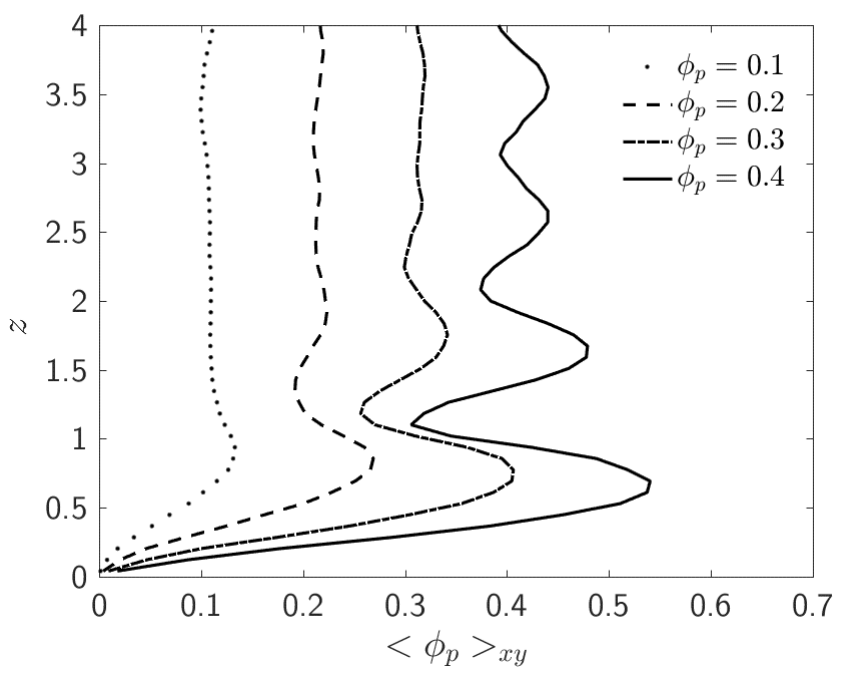

Figure 3: Wall normal profiles of the layer-average $\phi$

This wall induced perturbation of the particle volume fraction has been studied in literature for the case of freely sedimenting spheres in cylindrical containers [20, 19, 18, 17, 39]. Several correlations were proposed which are all limited to cylindrical coordinates and high packing fractions.

\subsubsection{Selection of an appropriate functional form}

The issue of formulating a proper functional form for $\langle\phi\rangle_{x z}$ (that will be referred to, for the sake of simplicity, simply as $\phi(z)$ in what follows) is notoriously difficult. This is best illustrated by considering the large number of different correlations proposed in literature for packed beds in cylindrical containers. One important property of $\phi(z)$ that can be inferred from Figure 3 is continuity. This property is not met by some of the correlations that have been proposed, for example, for packed beds in cylindrical containers (see for example [19]). Another characteristic is (as noticed before) the oscillatory nature of the local particle concentration as a function of the wall normal distance. Therefore, it is instructive to imagine $\phi(z)$ as a superposition of damped oscillating continuous functions. We next follow such a thought, and attempt to represent $\phi(z)$ in terms of a perturbation around an unperturbed value $\phi_{b}$.

$$
\begin{aligned}
& \phi(z)=\phi_{b}+\phi^{(0)}(z)+\phi^{(1)}(z)+\phi^{(2)}(z)+\phi^{(3)}(z)+\ldots \\
& \phi^{(n)}(z)=C_{n} J_{n}\left(\kappa_{n} z\right) \exp \left(\lambda_{n} z\right), \quad \forall n=0,1,2, \ldots
\end{aligned}
$$

Where $J_{n}$ is the $n$-th Bessel function of the first kind and the constants $C_{n}, \kappa_{n}$ and $\lambda_{n}$ are free parameters.

Therefore, $\phi(z)$ is the combination of a constant homogeneous particle volume fraction with a damped oscillation. Equations 17a can thus be rewritten as:

$$
\phi(z)=\phi_{b}+\sum_{n=0}^{\infty} C_{n} J_{n}\left(\kappa_{n} z\right) \exp \left(\lambda_{n} z\right)
$$

In some sense, equation 18 is a perturbation expansion of $\phi(z)$ around the homogeneous value $\phi_{b}$ that takes into account the presence of wall-induced inhomogeneities. In addition, one shall notice that only $J_{0}$ is not-vanishing at $z=0$, therefore implying $C_{0}=-\phi_{b}$.

Other correlations employ sine or cosine functions to represent the oscillations, together with a second order polynomial that models the near wall region (see for example [16]). Following such a purely empirical approach brings some uncomfortable characteristics: 
i The resulting profile is generally not continuous, at least in the first derivative. This may pose issues when evaluating wall-normal gradients of the particle volume fraction.

ii It does not split the unperturbed homogeneous bulk contribution from the perturbations due to the wall presence. Near wall expressions are generally carefully fitted for matching packed beds experiments (see [16]) and therefore, they fail in predicting the voidage in the case of more dilute particulate systems, e.g., as those encountered in fluidized bed applications.

iii Near-wall second order polynomials cannot provide a sufficiently good fit to our data, since we observe a turning point in the near-wall region (see Figure 3). Furthermore, we found better agreement when using Bessel functions compared to trigonometric functions (data not shown) for representing the oscillations.

iv Generally, such approach requires a larger number of fitting parameters (compared to an approach using Bessel functions) because two expressions need to be employed, i.e., one for each region.

Therefore, we approached the problem in a fairly general way by seeking a representation in series of exponentially damped Bessel functions for the perturbation terms. Such a choice (which was first adopted in a less general formulation by Mueller [20]) does not require the adoption of ad-hoc functions for the near wall region. Remarkably, the use of a Bessel function has been discouraged in literature due to the lack of experimental data that indicates a turning point in the near wall region [15]. However, since we do observe a turning point, a Bessel function-based approach has significant appeal. Finally, one can speculate that the contribution of higher order terms in equation 18 may be used to suppress the turning point of the voidage profile in case of curved walls. In such a way Bessel function-based approaches could be made fit to represent voidage profiles in cylindrical containers as well.

\subsubsection{Evaluation of the free parameters}

Before attempting to evaluate the other free parameters (we found $C_{0}=-\phi_{b}$ already), we have to draw some considerations (and assumptions) about their functional dependence. It is expected, by observing Figure 3, that all the parameters are somehow depending on $\phi_{b}$. Specifically:

i $C_{n}$ represents the amplitude, which scales with $\phi_{b}$ same as $C_{0}$.

ii $\kappa_{n}$ is a wave number, which increases with increasing $\phi_{b}$, and must saturate when approaching the close packing limit. In fact, from $\phi_{p}=0.1$ to $\phi_{p}=0.4$ the oscillatory nature of $\phi(z)$ becomes more evident.

iii $\lambda_{n}$ is a decay constant of the perturbation and it is therefore expected to be a decreasing function of $\phi_{b}$. This is since the spheres are closer in situations characterized by a higher packing fractions, thus transmitting the perturbation to their neighbours more effectively.

We also assume that the free parameters are independent of $z$ or, in other words, that the arguments of the $J_{n}$ and exp functions are linear in $z$. Finally, we aim to establish if, like for the correlation of Mueller [20], the free parameters are depending upon the vertical domain length $L_{z}$. However, following our approach $\phi(z)$ should not depend explicitly on $L_{z}$ (like in Mueller [20]), but it is concealed in the definition of the bulk particle volume concentration $\phi_{b}$, i.e. smaller $L_{z}$ implies larger $\phi_{b}$ due to mass conservation.

In order to evaluate the free parameters, we performed a different set of purely DEM-based simulations using LIGGGHTS $^{\circledR}$. These simulations used a similar configuration as our main study, but with a dimensionless distance $L_{z}=12$ between the two walls (therefore, 50\% larger than our main study). We probed values of $\phi_{p}$ between $\phi_{p}=0.05$ and $\phi_{p}=0.65$ with increments of $\Delta \phi_{p}=0.05$. We first fitted the resulting $\phi(z)$ profiles with equation $17 \mathrm{a}$, and subsequently we fitted the obtained parameters with properly chosen functions of $\phi_{b}$. This resulted in the following correlation for the first order perturbed solid volume fraction $\phi^{(0)}(z)$ :

$$
\begin{aligned}
& \phi^{(0)}(z)=-\phi_{b} J_{0}\left(\kappa_{0} z\right) \exp \left(-\lambda_{0} z\right) \\
& \kappa_{0}=0.875+8.550 \sqrt{\phi_{b}} \\
& \lambda_{0}=0.461+0.409 \sin \left(7.421 \phi_{b}+0.327\right)
\end{aligned}
$$




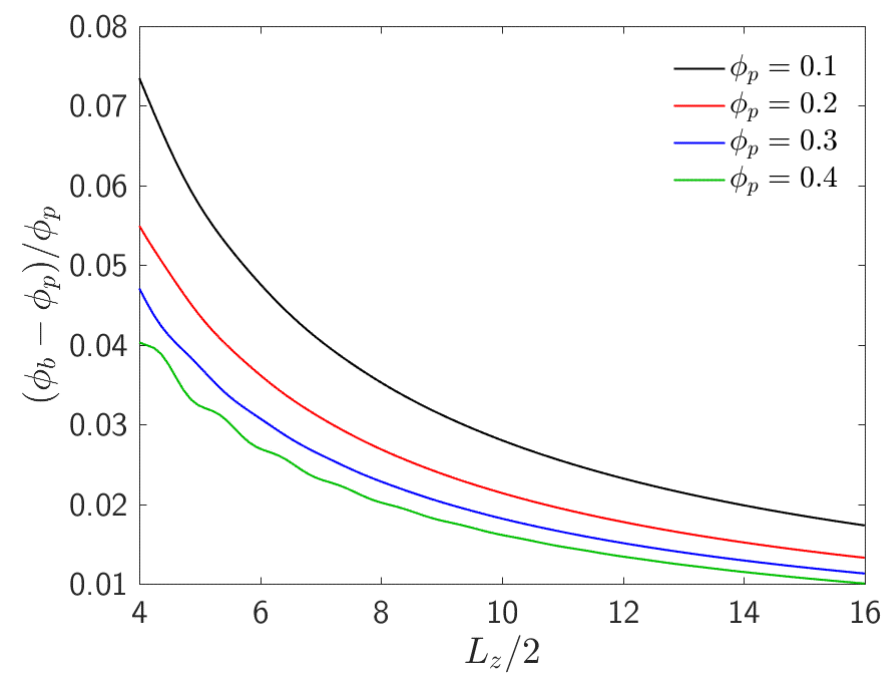

Figure 4: Relative deviation of the bulk particle concentration $\phi_{b}$ with respect to the average particle concentration $\phi_{p}$ for different values of $L_{z}$.

\subsubsection{Evaluation of the bulk particle volume concentration - finite domain effects}

Our formulation based on the perturbation expansion 17a allows a to easily generalize our correlation to the case of finite domains where walls are separated by a distance $L_{z}$. The average particle concentration on a volume $V$ can be defined as:

$$
\phi_{p}=\frac{1}{V} \sum_{p=1}^{N} m_{p}=\frac{1}{L_{z}} \int_{0}^{L_{z}} \phi(z) \mathrm{d} z
$$

Clearly, $\lim _{L_{z} \rightarrow \infty} \phi_{b}=\phi_{p}$ must hold. In other words, since $\phi_{b}$ can be also defined asymptotically from $\lim _{L_{z} \rightarrow \infty} \phi(z)=$ $\phi_{b}$, the particle concentration measured in the bulk differs from the (global) average particle concentration.

Substituting the first order perturbation leads to:

$$
\phi_{p}=\frac{1}{L_{z}} \int_{0}^{L_{z}}\left[\phi_{b}-\phi_{b} J_{0}\left(\kappa_{0} z\right) \exp \left(\lambda_{0} z\right)\right] \mathrm{d} z
$$

Which leads to the following integral equation for the bulk concentration as a function of $L_{z}$ :

$$
\phi_{b}\left(L_{z}\right)=\phi_{p}\left[1-\frac{1}{L_{z}} \int_{0}^{L_{z}} \frac{1}{L_{z}} J_{0}\left(\kappa_{0}\left(\phi_{b}\right) z\right) \exp \left(\lambda_{0}\left(\phi_{b}\right) z\right) \mathrm{d} z\right]^{-1}
$$

Equation 22 is solved iteratively for different values of the domain size ranging from $L_{z}=8$ (which is the value used in our main study) to $L_{z}=32$. As shown in Figure 4, the effect of a finite domain size is relatively small (i.e., a few percent at most) for the values of $L_{z}$ we considered. Also, the domain size effect decreases to a first approximation exponentially with increasing $L_{z}$. Therefore, we do not provide any expression for the bulk particle concentration since in most cases of practical interest the cell size of an EL or EE simulation is sufficiently large (compared to the particle diameter) to accept the approximation $\phi_{b} \approx \phi_{p}$.

However, Figure 4 provides a measure of the error committed in using finite domains to study wall effects in dense suspensions or, more generally speaking, in granular systems consisting of monodispersed spheres. 


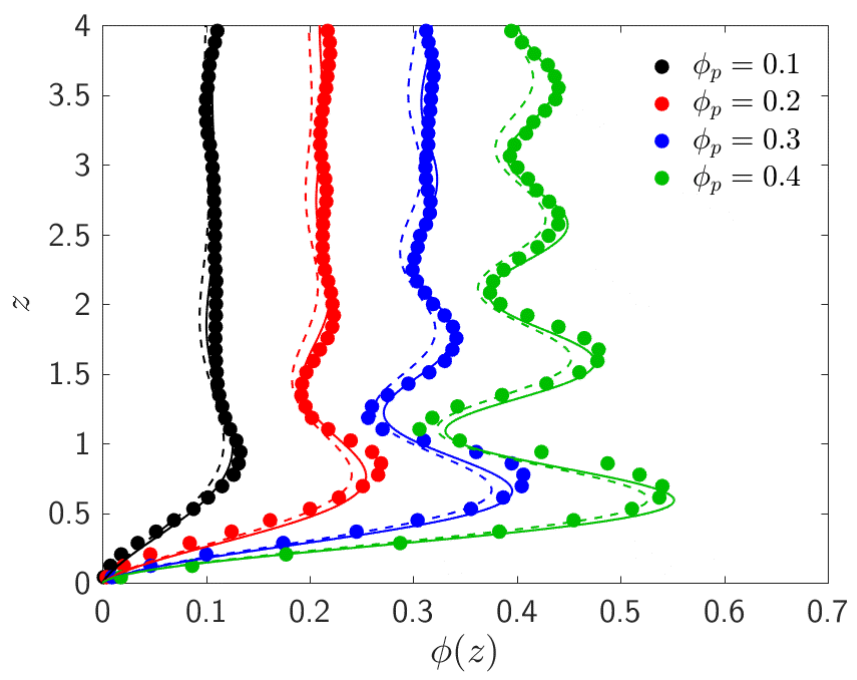

Figure 5: Plot of $\phi(z)$ for four different values of the average particle concentrations. Dots are data from simulations and lines are the first order correlation described by equation $19 \mathrm{a}$. Continuous lines are obtained calculating $\phi_{b}$ from equation 22 while for dashed lines we took $\phi_{b}=\phi_{p}$. The average deviation of the correlation from simulations is: $10.5 \%\left(\phi_{b}=0.1\right), 9.6 \%\left(\phi_{b}=0.2\right), 5.9 \%\left(\phi_{b}=0.3\right)$ and $5.6 \%\left(\phi_{b}=0.4\right)$ when $\phi_{b}=\phi_{p}$ (dashed lines). The error decreases when $\phi_{p}$ is computed correctly (continuous lines): $7.1 \%\left(\phi_{p}=0.1\right), 6.2 \%\left(\phi_{p}=0.2\right), 3.9 \%\left(\phi_{p}=0.3\right)$ and $4.2 \%\left(\phi_{p}=0.4\right)$.

\subsubsection{Validation of the proposed correlation}

Finally, we compared $\phi(z)=\phi_{b}+\phi^{(0)}(z)$ with results from our main study (see Figure 5). We stress that data presented in Figure 5 differ from with the data used to determine the free parameters. The two set of simulations are completely separated and performed using a different domain size. Nevertheless, we found excellent agreement between the correlation and the data used for the main study, showing that the domain size is not a critical parameter for the coefficients (at least, in the relatively large domains we investigated) but it is implicit in the definition of $\phi_{b}$ when using equation 22. Furthermore, we notice that the error is decreasing with increasing packing fraction. This is in agreement with the previous considerations and in particular with the interpretation of Eqn. 17a as a perturbation around the homogeneous value. In fact, the leading term $\phi^{(0)}(z)$ represents the main contribution to the perturbation since it is the one which carries the wall main disturbance (i.e., the one that sets $\phi(z)$ to zero). In other words, additional terms in the expansion can be viewed as contribution due to the number of ways in which particles can be distributed. The higher the order, the more randomness is allowed.

Even if more accurate approximations could be obtained by including higher order contributions, the fitting procedure for the resulting free parameters poses several challenges. Specifically, we found that it becomes increasingly difficult to identify a recognizable functional dependence on $\phi_{b}$. We have therefore suppressed the discussion of these dependencies, and remain with the first two contributions shown on the right hand side of Eqn. 17a. We note in passing that additional terms may become relevant in the case of curved walls. This is since these terms can be potentially used to suppress the turning point in the near wall region.

While there exist several correlations for the special case of packed beds bounded by cylindrical walls, no expression have been proposed for somewhat more dilute systems or flowing suspensions. Therefore, a comparison of our data with existing correlations would lack meaning. We have therefore suppressed such a comparison, also for the reasons exposed in 4.1.1.

\subsection{Wall normal velocity and temperature profiles}

The perturbation to the particle volume fraction shown in Figure 3 and described by means of equation 17a, gives rise to statistically inhomogeneous velocity and temperature fields as a function of the wall normal distance. Furthermore, considering the profiles shown in Figures 6 and 7, we can conclude that most of the boundary layer effects are concentrated in the region $z=[0,1]$, and they are therefore limited to the first particle layer. 


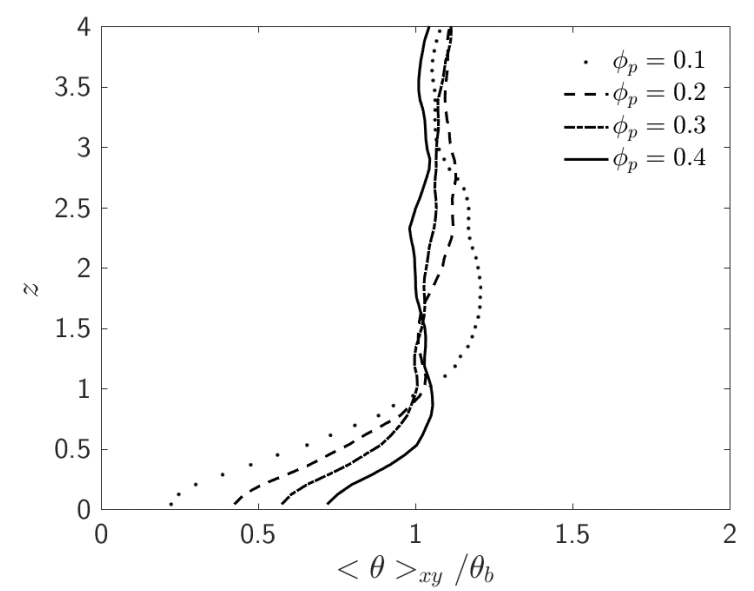

(a) $\operatorname{Re}=100$

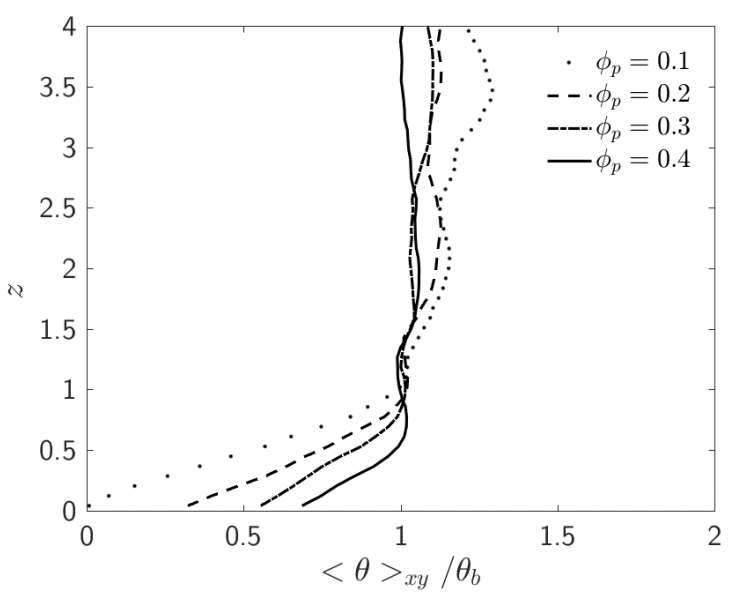

(c) $\operatorname{Re}=300$

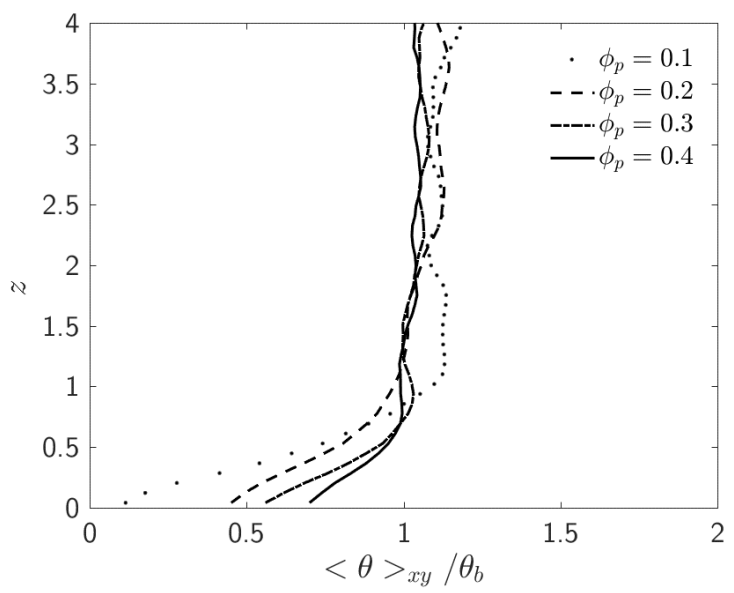

(b) $R e=200$

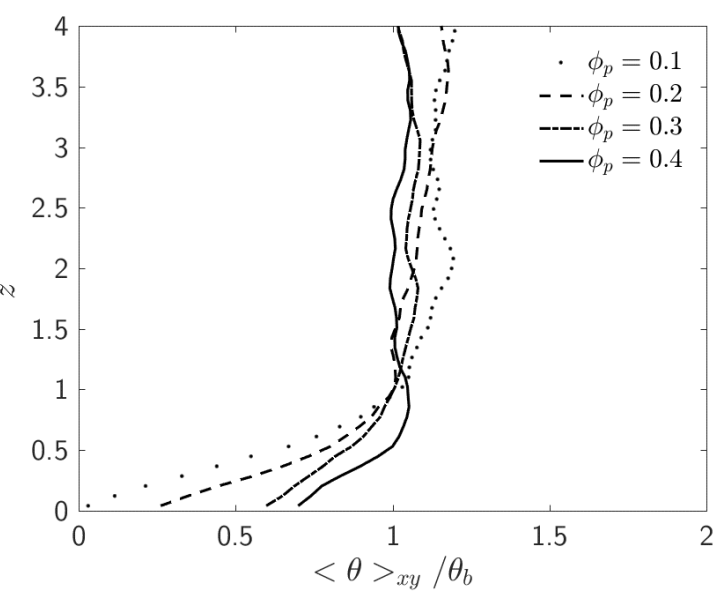

(d) $R e=400$

Figure 6: Wall normal profiles of $\theta . R e=100$ (6a), $R e=200(6 \mathrm{~b}), R e=300(6 \mathrm{c}), R e=400$ (6d). 


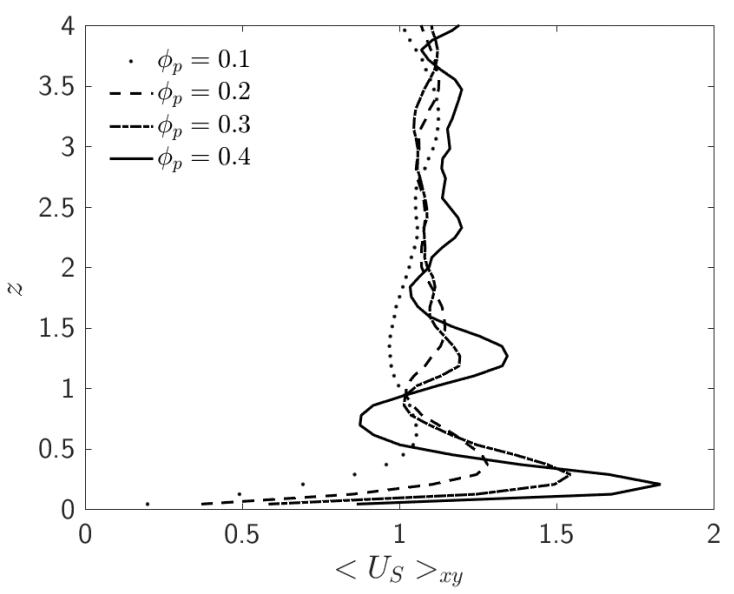

(a) $R e=100$

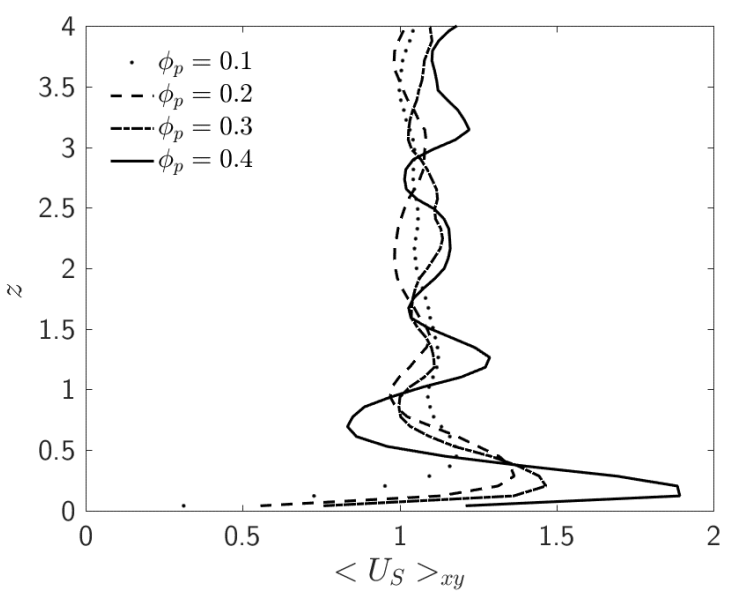

(c) $R e=300$

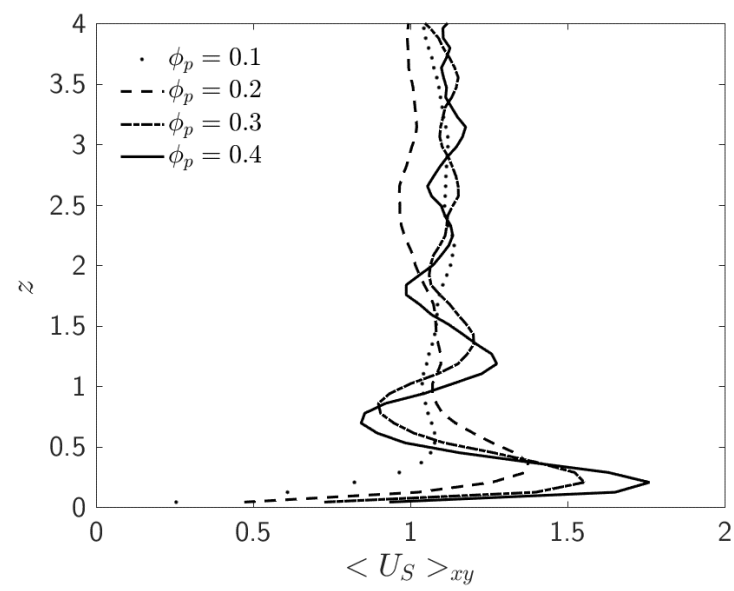

(b) $R e=200$

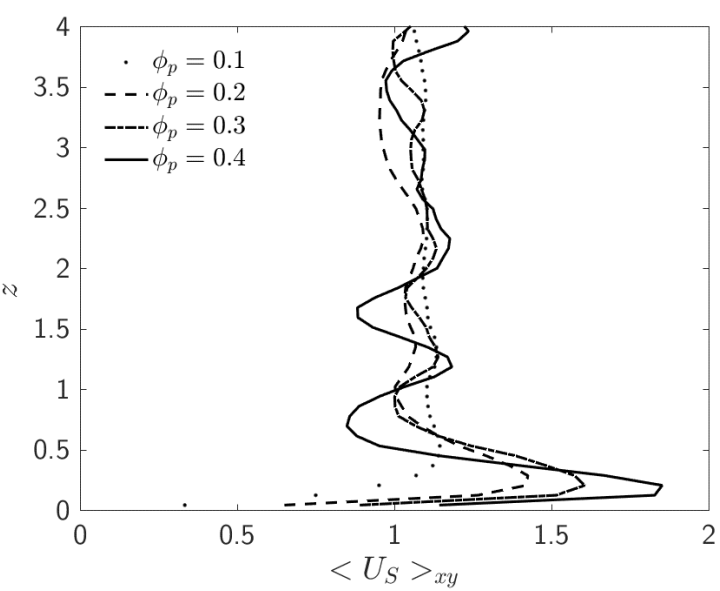

(d) $R e=400$

Figure 7: Wall normal profiles of $U_{s}$ (streamwise direction). $R e=100$ (7a), $\operatorname{Re}=200$ (7b), $\operatorname{Re}=300$ (7c), $\operatorname{Re}=400$ (7d).

This is especially true for the layer averaged temperature field $\theta$ shown in Figure 6. In addition, we conclude that the effect of the Reynolds number on the velocity profiles is - to a first approximation - negligible with respect to the effect of the bulk particle volume fraction $\phi_{b}$ (Figure 7). Increasing the fluid speed is therefore equivalent to uniformly scale the velocity field.

Unlike the velocity field, the temperature field seems to be (weakly) affected by the Reynolds number. However, this can be interpreted in terms of the Peclet number as for low Pe the diffusive nature of heat transport tends to produce more uniform profiles (remember that we are using adiabatic walls). In contrast, for high $P e$, the heat is quickly removed by advection in the near wall region, due to the higher fluid speed in this region.

Therefore, it is natural to next attempt defining wall normal temperature and velocity fields $\theta^{\prime}(z)=\left\langle\langle\theta\rangle_{x z}\right\rangle_{R e}$ and $u^{\prime}(z)=\left\langle\left\langle U_{s}\right\rangle_{x z}\right\rangle_{R e}$. This is equivalent to neglecting the effect of the Reynolds number on the wall normal profiles.

As shown in Figures 6 and 7, the presence of the discrete phase gives rise to a particle induced thermal boundary field $z<z_{\ell, \theta}$ and a particle induced dynamic boundary field $z<z_{\ell, u}$ such that to a first approximation one can accept:

$$
\theta^{\prime}(z)= \begin{cases}\theta_{\ell}^{\prime}(z) & \text { if } z \leq z_{\ell, \theta} \\ 1 & \text { if } z>z_{\ell, \theta}\end{cases}
$$




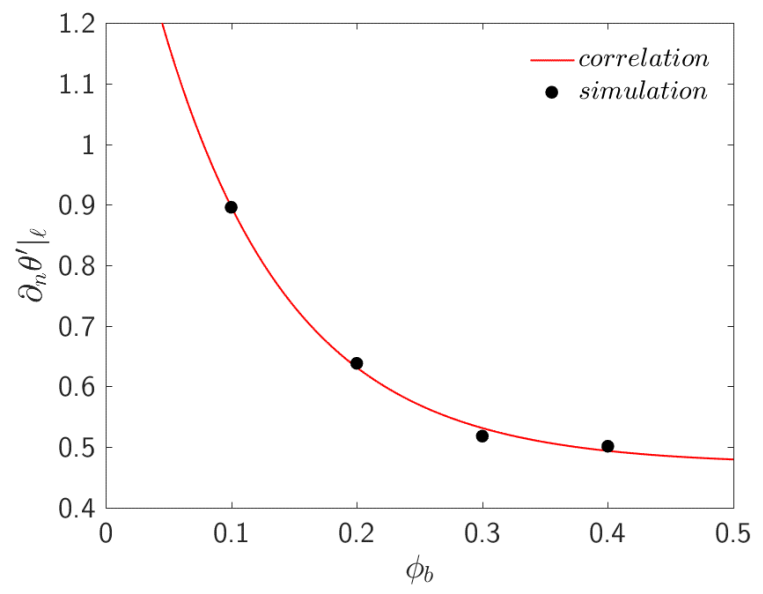

(a)

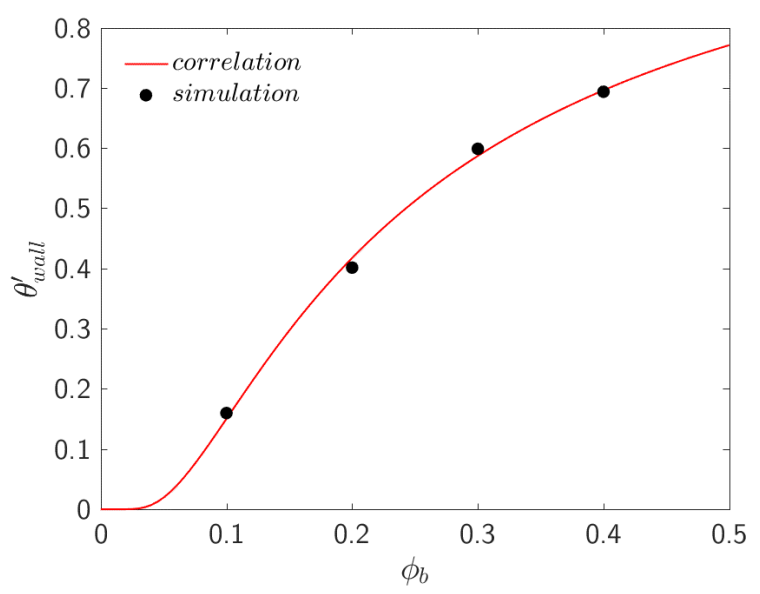

(b)

Figure 8: Comparison of equations 26a (a) and 27a (b) with simulation data.

$$
u^{\prime}(z)= \begin{cases}u_{\ell}^{\prime}(z) & \text { if } z \leq z_{\ell, u} \\ 1 & \text { if } z>z_{\ell, u}\end{cases}
$$

Where $\theta_{\ell}^{\prime}(z)$ and $u_{\ell}^{\prime}(z)$ represent the perturbation to the homogeneous bulk field induced by the wall boundaries.

Instead of trying to accurately reconstruct these functions, we next isolate the most physically interesting quantities and use them to build an approximate profile.

\subsubsection{Wall temperature and wall-normal temperature gradient}

It is quite remarkable that despite the fact that we used Neumann boundary conditions at the walls, Figure 6 clearly shows the existence of a temperature gradient in the boundary layer. Consequently, particles located within this region experience a mean fluid temperature gradient that superposes the mean fluid-particle temperature difference. The mean temperature gradient is caused by a heat flux pointing from the bulk towards the walls, which is compensated by convective heat transport in the wall-parallel direction.

The gradient in the wall normal direction can be quantified by computing an average gradient in the wall near region:

$$
\left.\partial_{z} \theta^{\prime}\right|_{\ell}=z_{\ell, \theta}^{-1} \int_{0}^{z_{\ell, \theta}} \partial_{z} \theta^{\prime} \mathrm{d} z=z_{\ell, \theta}^{-1}\left(\theta^{\prime}\left(z_{\ell, \theta}\right)-\theta^{\prime}(0)\right)=z_{\ell, \theta}^{-1}\left(1-\theta_{\text {wall }}^{\prime}\right)
$$

We propose the following expression for the the wall normal temperature gradient $\left.\partial_{z} \theta^{\prime}\right|_{\ell}$ :

$$
\begin{aligned}
& \left.\partial_{z} \theta^{\prime}\right|_{\ell}\left(\phi_{b}\right)=C_{1}+C_{2} \exp \left(C_{3} \phi_{b}\right) \\
& C_{1}=0.471, \quad C_{2}=1.131, \quad C_{3}=-9.763 .
\end{aligned}
$$

Figure 8a shows that expression 26a yields indeed a good approximation to our data.

Another quantity of practical interest is the temperature perturbation at the wall $\theta_{\text {wall }}^{\prime} \leq 1$ which is induced by the dispersed phase. Again, we use our data to derive a correlation:

$$
\theta_{\text {wall }}^{\prime}\left(\phi_{b}\right)=C_{1} \exp \left(\frac{C_{2}}{\phi_{b}}\right)
$$




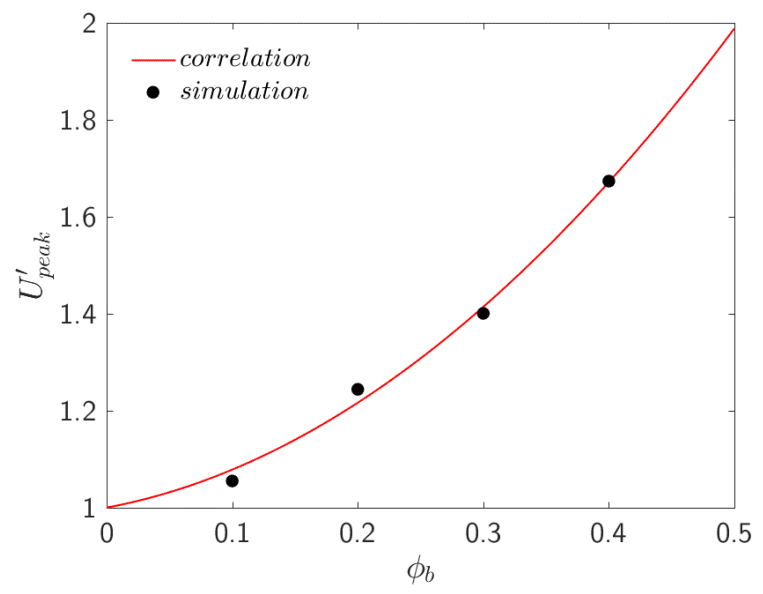

(a)

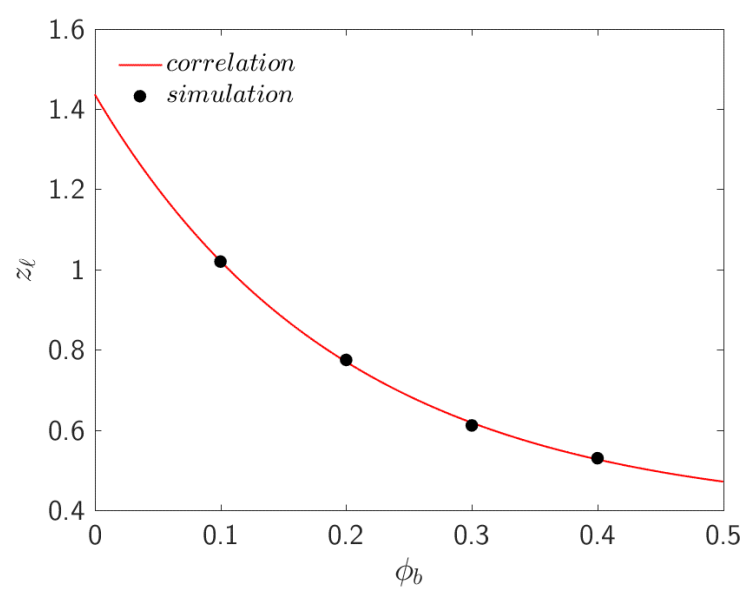

(b)

Figure 9: Comparison of equations 28a (a) and 29a (b) with simulation data

$$
C_{1}=1.160, \quad C_{2}=-0.204
$$

As shown in Figure 8b, the function we used is respecting physically-meaningful limiting conditions (i.e., $\lim _{\phi_{b} \rightarrow 0} \theta_{\text {wall }}^{\prime}=$ 0 , and $\max \left(\theta_{\text {wall }}^{\prime}\right)<1$, as well as approximates our data convincingly well.

\subsubsection{Wall shear stress}

Regarding the velocity field, one would generally be interested in quantifying the magnitude and height of the peak value $u_{\text {peak }}^{\prime}$ as well as the normalized wall normal shear stress $\tau_{x z, w}$. The latter is proportional to $\left.\partial_{z} u^{\prime}\right|_{w}$, i.e., the velocity gradient at the wall.

As for the wall temperature and the temperature gradient, we express such quantities as a function of the bulk particle volume fraction $\phi_{b}$ :

$$
\begin{aligned}
& u_{\text {peak }}^{\prime}=1+C_{1} \phi_{b}+C_{2} \phi_{b}^{2} \\
& C_{1}=0.486, \quad C_{2}=2.983 \\
& z_{\text {peak }}=C_{1}+C_{2} \exp \left(C_{3} \phi_{b}\right) \\
& C_{1}=0.153, \quad C_{2}=1.151, \quad C_{3}=-11.749
\end{aligned}
$$

We note in passing that equation 28a satisfies the $\operatorname{limit}_{\lim _{\phi_{b} \rightarrow 0}} u_{\text {peak }}^{\prime}=1$, which reflects the obvious fact that no particle induced velocity boundary layer exists in the absence of particles. In addition, we recall that the dimensional wall distance is given by $z^{\star}=z d_{p}$. Therefore, no peak will be observed for infinitesimally small particles.

Equations $28 \mathrm{a}$ and $29 \mathrm{a}$ can be used to evaluate $\left.\tau_{x z}\right|_{w}$. This can be done by representing $u^{\prime}$ in Taylor series at $z=0$ :

$$
u^{\prime}(z)=\left.\sum_{k=0}^{\infty} \frac{1}{k !} \frac{\partial^{k} u^{\prime}}{\partial z^{k}}\right|_{z=0} z^{k}
$$

Our simulations show that the function $u^{\prime}(z)$ exhibit an almost parabolic behaviour in the interval $z \in\left[0, z_{\text {peak }}\right]$. Therefore, we seek for a closed expression for $\left.\tau_{x z}\right|_{w}$ by retaining only terms up to $O\left(z^{2}\right)$ in Eqn. 30. Considering the no-slip condition at the wall, we arrive at 


$$
u^{\prime}(z)=u^{\prime}(0)+\left.\partial_{z} u^{\prime}\right|_{0} z+\left.\frac{1}{2} \frac{\partial^{2} u^{\prime}}{\partial z^{2}}\right|_{z=0} z^{2}+O\left(z^{3}\right)=\left.R e^{-1} \tau_{x z}\right|_{w} z+\left.\frac{1}{2} \frac{\partial^{2} u^{\prime}}{\partial z^{2}}\right|_{z=0} z^{2}+O\left(z^{3}\right)
$$

This has to be provided with the condition that $z_{\text {peak }}$ is a critical point, and should satisfy a concavity condition:

$$
\left.\frac{\partial u^{\prime}}{\partial z}\right|_{z=z_{\text {peak }}}=0, \quad \partial_{z z} u^{\prime}<0 \quad \forall z \in\left[0, z_{\text {peak }}\right]
$$

Finally, we obtain the following equation for $u^{\prime}(z)$ :

$$
u^{\prime}\left(z^{\prime}\right)=u_{\text {peak }}^{\prime} z^{\prime}\left(2-z^{\prime}\right), \quad z^{\prime}=z / z_{\text {peak }}
$$

This ultimately leads to:

$$
\left.\tau_{x z}\right|_{w}=\frac{2}{R e} \frac{u_{\text {peak }}^{\prime}}{z_{\text {peak }}}=\frac{2}{\operatorname{Re}} \frac{u_{\text {peak }}^{\prime}}{z_{\text {peak }}}=\frac{2}{\operatorname{Re}} \frac{1+0.486 \phi_{b}+2.983 \phi_{b}^{2}}{0.153+1.151 \exp \left(-11.749 \phi_{b}\right)}
$$

Equation 34 allows to evaluate the (normalized) wall shear stress induced by the fluid-particle system as a function of the bulk particle concentration and the Reynolds number only.

\subsection{Benchmarks for the Nusselt number prediction}

Since we established that wall induced perturbations are mostly relevant within $1 d_{p}$ from the walls, it makes sense to compare the Nusselt number experienced by bulk particles (i.e., particle located beyond $1 d_{p}$ from a wall) with existing correlations. In particular, we compare our result with the correlation from Deen et al. [33]:

$$
N u_{\text {Deen }, i}=\left(7-10 \phi_{f}+5 \phi_{f}^{2}\right)\left(1+0.17 \operatorname{Re}_{i}^{0.2} \operatorname{Pr}^{1 / 3}\right)+\left(1.33-2.31 \phi_{f}+1.16 \phi_{f}^{2}\right) \operatorname{Re}_{i}^{0.7} \operatorname{Pr}^{1 / 3}
$$

Notice that we used $\phi_{f}=1-\bar{\phi}\left(\boldsymbol{x}_{i}\right)$ and $\operatorname{Re} e_{i}=\operatorname{Re} \bar{\phi}\left(\boldsymbol{x}_{i}\right) \widetilde{U_{s}}\left(\boldsymbol{x}_{i}\right)$ in the above expression. As already pointed out in our previous work [12], expression 35 is not consistent with PU-EL since the cup-mixing temperature was used. This temperature is different from the temperature available in a PU-EL simulation. A correction was proposed by Sun et al. [32] that modifies the above correlation to be consistent with PU-EL:

$$
N u_{D S, i}=\frac{N u_{D e e n, i}}{1-1.6 \bar{\phi}\left(\boldsymbol{x}_{i}\right)\left(1-\bar{\phi}\left(\boldsymbol{x}_{i}\right)\right)-3 \bar{\phi}\left(\boldsymbol{x}_{i}\right)\left(1-\bar{\phi}\left(\boldsymbol{x}_{i}\right)\right)^{4} \exp \left(-\operatorname{Re}_{i}^{0.4} \bar{\phi}\left(\boldsymbol{x}_{i}\right)\right)}
$$

In Figure 10, we plot the calculated particle-based Nusselt number over the prediction from equation 36 . The comparison shows that our methodology is fully capable of reproducing the results of Deen et al. [33] and Sun et al. [32], which are given in terms of an average, and not particle-based, Nusselt number. Discrepancies are observed for (i) high values of $N u$ (which are, however, outside the range of validity of correlation denoted as $N u_{D S}$ ), and (ii) for $\rho=2$ (see Figure 10a). The latter disagreement can be explained by noticing that, as addressed in our previous work [12], for such filter size local inhomogeneities in the particle bed become relevant. Overall, the agreement can be considered satisfactory and details about average deviation are summarized in Table 2 .

Unlike the approach used in the derivation of $N u_{\mathrm{DS}}$, our approach allows to evaluate the dispersion of the particle based quantities around the average value (note, only the latter is provided by the correlation). As shown in Table 2 , the deviation is very high for small filter sizes but seems to approach a constant value of $\sigma\left(N u_{i} / N u_{\mathrm{DS}, i}\right) \approx 0.36$ as the filter size is increased. Thus, fluctuations around the mean are substantial, and should be potentailly considered in closures for PU-EL models. 


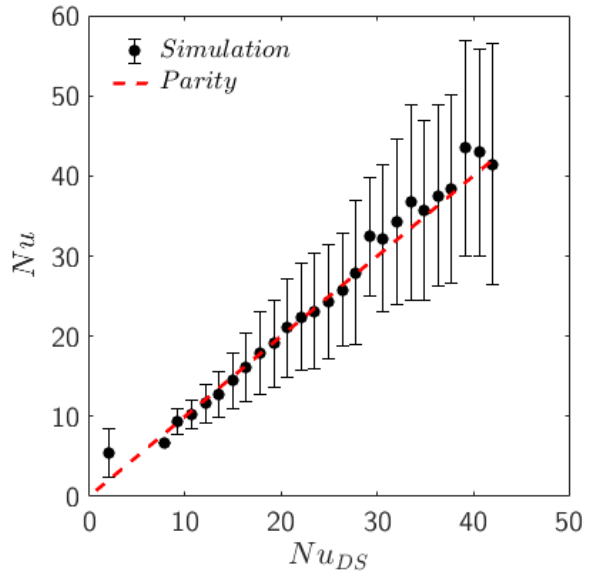

(a) $\varrho=2$

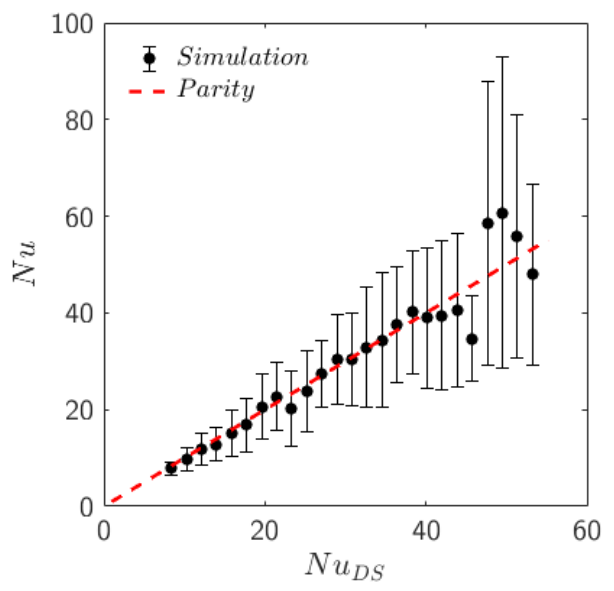

(c) $\varrho=4$

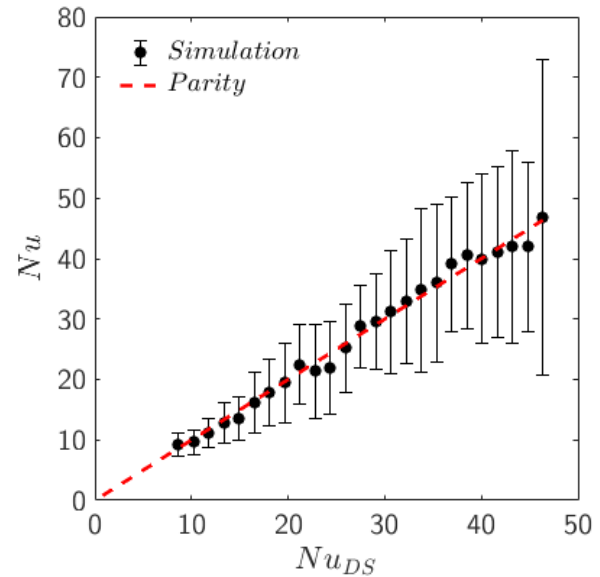

(b) $\varrho=3$

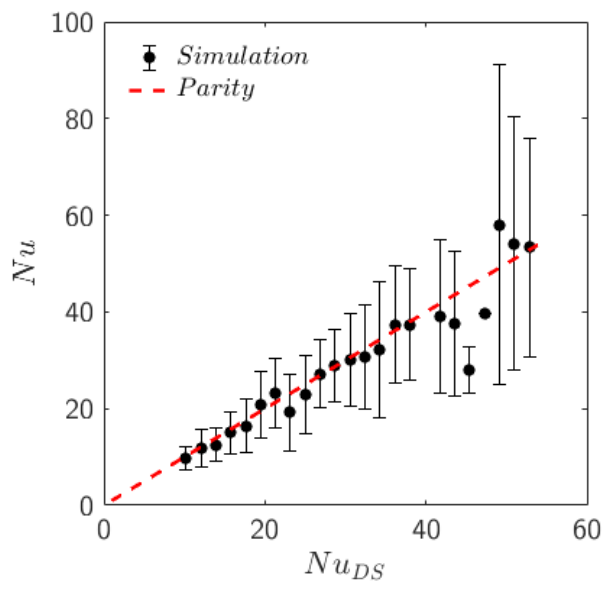

(d) $\varrho=5$

Figure 10: Parity plot showing the binned particle based Nusselt number compared with the predicion of Deen et al. and Sun et al. Error bars extend to two standard deviations. Different panels refer to different values of the dimensionless filter size $\varrho$. 
Table 2: Average relative error and standard deviation of the particle based Nusselt numbers from values predicted by equation $36 . N u_{\mathrm{DS}, i}$ is used as a short hand for $N u_{\mathrm{DS}}\left(\bar{\phi}(\boldsymbol{x})_{i}, R e_{i}\right)$.

\begin{tabular}{ccc}
\hline$\varrho$ & $\left\langle\frac{N u_{i}}{N u_{\mathrm{DS}, i}}\right\rangle$ & $\sigma\left(\frac{N u_{i}}{N u_{\mathrm{DS}, i}}\right)$ \\
\hline 2 & 1.37 & 0.87 \\
3 & 0.99 & 0.34 \\
4 & 0.98 & 0.36 \\
5 & 0.95 & 0.36 \\
\hline
\end{tabular}

\subsection{Wall corrections to the drag coefficient and Nusselt number}

In order to quantify the effect of wall boundaries on the drag coefficient and Nusselt number, we collected the statistics using the binning approach that is described in one of our previous studies [12].

As a result, we observed that (as for the wall normal velocity and temperature profiles) the dependence on the Reynolds number can be easily dropped using the following scaling:

$$
F\left(z, \phi_{b}, R e\right)=\zeta_{F}\left(z, \phi_{b}\right) F_{b}\left(z, \phi_{b}, R e\right)
$$

Where $F_{b}\left(z, \phi_{b}, R e\right)$ is the bulk drag force which is calculated using the approach shown in Section 2.4. Therefore, such bulk value represents the homogenous drag calculated using $\phi_{b}$ rather than $\phi_{p}$.

The fact that $\zeta_{F}\left(z, \phi_{b}\right)$ is not a function of the Reynolds number comes from our data analysis and is in agreement with the previous study of the near wall behaviour of the velocity profiles. Therefore, in order to evaluate $\zeta_{F}\left(z, \phi_{b}\right)$, we consider particles from simulations with different Reynolds numbers in the bins having the same wall normal distance. This strategy allows us to collect more robust statistics on mean exchange coefficients.

In order to further prove that the decomposition expressed in equation 37 is valid, we compared results obtained from the current set of simulations with results from one of our previous studies [40]. This latter work featured different particle configurations, but a fixed Reynolds number of 100. The total number of particle is almost the same for both studies. Figure 11 shows that decomposition 37 leads to an excellent agreement between the two studies, therefore supporting our argument that the Reynolds number does not play an essential role when determining wall profiles.

The data we present in this section are therefore a combination of data from both studies, so that for each value of $\phi_{b}$ we use data from simulations involving approximately 4,000 particles.

Figure 12 shows results for $\zeta_{F}\left(z, \phi_{b}\right)$. All wall normal profiles have a maximum corresponding to the first particle layer $(z=0.5)$ and a minimum develops with increasing $\phi_{b}$. It can also be seen that the intensity of the perturbations becomes larger with larger $\phi_{b}$, which is probably related to the stronger perturbation in the particle concentration. The effect of the filter size $\rho$ is not existing, hinting to the fact that filtered fluid velocities are almost independent of $\rho$.

Values of $\zeta_{F}\left(z, \phi_{b}\right)$ are tabulated in tables A.3 and A.4. The latter are meant to support the implementation of wall corrections to be used in PU-EL-based simulation models.

We then extend the same approach to the study of Nusselt number profiles by defining:

$$
N u\left(z, \phi_{b}, R e\right)=\zeta_{N u}\left(z, \phi_{b}\right) N u_{b}\left(z, \phi_{b}, R e\right)
$$

Results for $\zeta_{N u}\left(z, \phi_{b}\right)$ are plotted in Figure 13 and tabulated in Tables A.5 and A.6 in the Appendix. The results indicate that corrections to the Nusselt number are in general strong as compared to that for the drag. This can be tentatively explained by the combined effect of flow (i.e., the velocity gradient) and the abnormal temperature distribution experienced by wall-near particles.

Futhermore, the filter size $\rho$ has a significant effect on the Nusselt number correction. This effect is extreme for the wall-closest particles, i.e., that located at $z=0.5$. The enormous sensitivity to $\rho$ observed for the corrections to the Nusselt number is in contrast to the correction for the drag coefficient. The latter are almost insensitive to the filter size. 


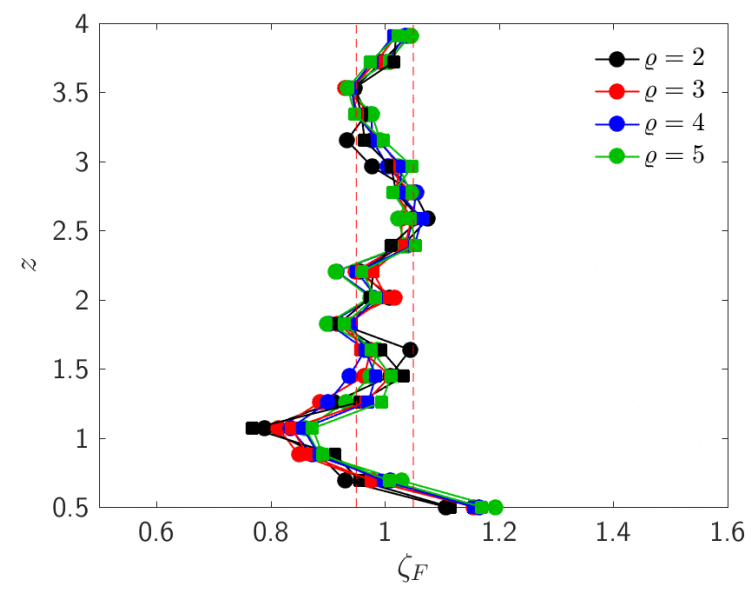

(a) $\phi_{b}=0.3$

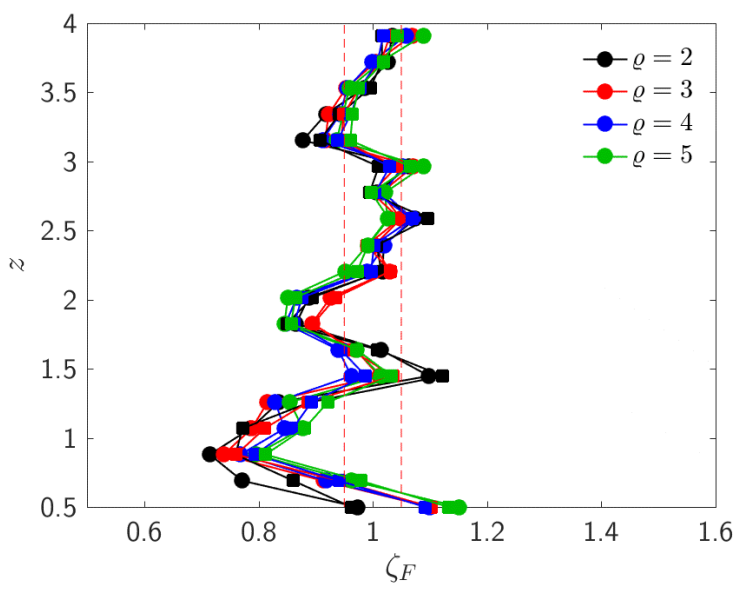

(b) $\phi_{b}=0.4$

Figure 11: Comparison between results for $\zeta_{F}\left(z, \phi_{b}\right)$ from the this work (disks) and our previous work with $R e=100$ (squares). Red dashed lines represent deviations of $\pm 5 \%$ from unity (i.e., the bulk value). The same comparison carried out for $\zeta_{N u}\left(z, \phi_{b}\right)$ and for different values of $\phi_{b}$ leads to a similar agreement between the two studies and is included in Appendix A.

We speculate that the origin of this difference is the abnormal temperature distribution close to the wall: Depending on the filter size, a different filtered temperature is experienced by individual particles. Specifically, smaller filter sizes $\rho$ will lead to lower values for $\langle\theta\rangle_{x y}$ as seen in Figure 6. This will lead to a larger difference $\theta_{p}-\langle\theta\rangle_{x y}$. In simple words, a smaller filter size (or a smaller grid size in PU-EL models) results in wall-near particles experiencing a different (in the context of our study a cooler) fluid temperature. Thus, for a given fluid-particle heat flux, the Nusselt number must decrease for a decrease in the filter size in case particles are located in the 'temperature boundary layer' observed in Figure 6. In Figure 13 we observe exactly this trend for the correction of the Nusselt number and for $\phi>0.1$. In case particles are outside of the 'temperature boundary layer', the above argument on the filter size effect is expected to break down. Also this fact is observable in Figure 13 when considered the data for $z>1.2$. Finally, one should mention that for dilute systems (exemplify by $\phi>0.1$ in our present study) the 'temperature boundary layer' is only weakly pronounced. This is not directly observable from Figure 6, however, a fact since in Figure 6 the temperature profile is scaled with the bulk value. The latter is strongly decreasing for decreasing particle concentrations. Hence, we do not observe noticeable corrections to the Nusselt number for $\phi=0.1$ in Figure 13 . 


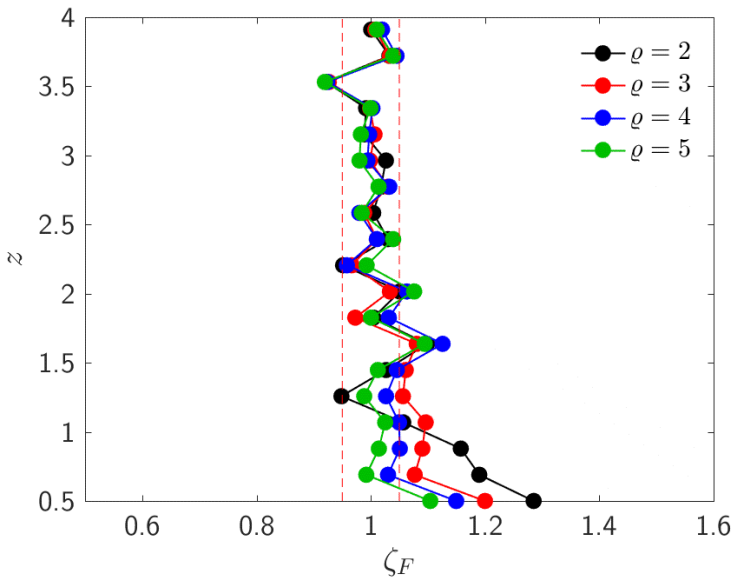

(a) $\phi=0.1$

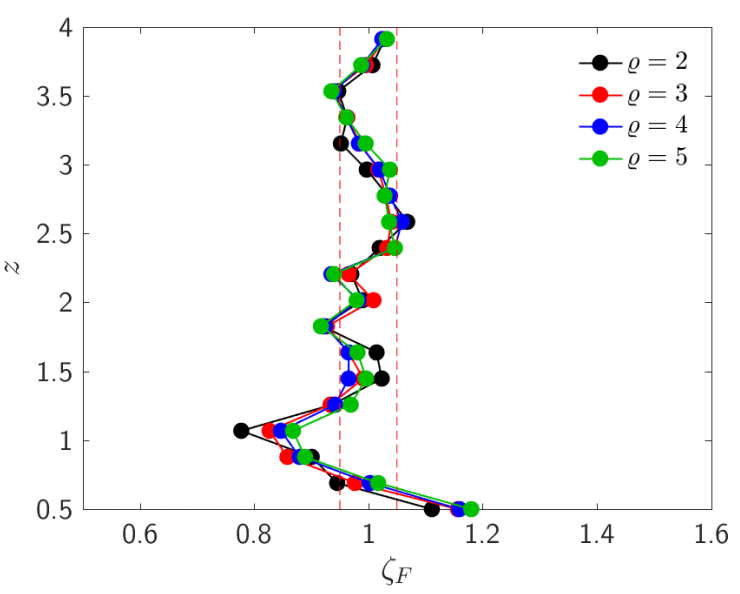

(c) $\phi=0.3$

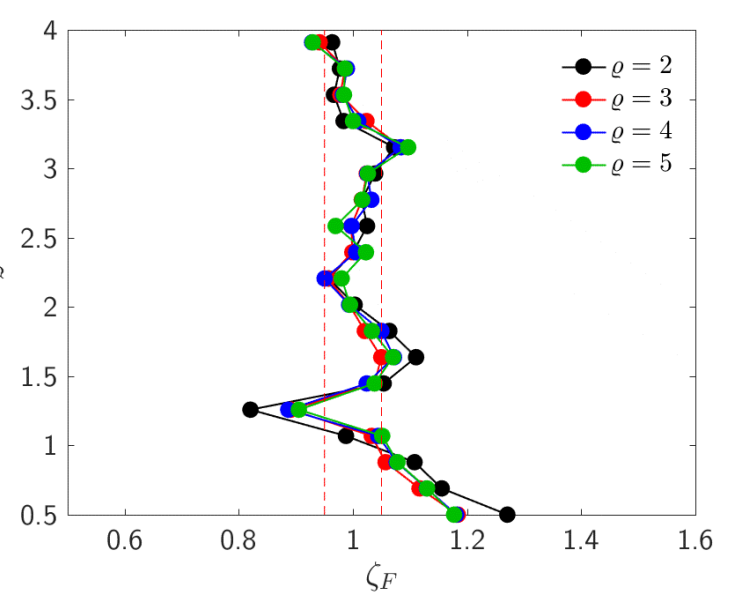

(b) $\phi=0.2$

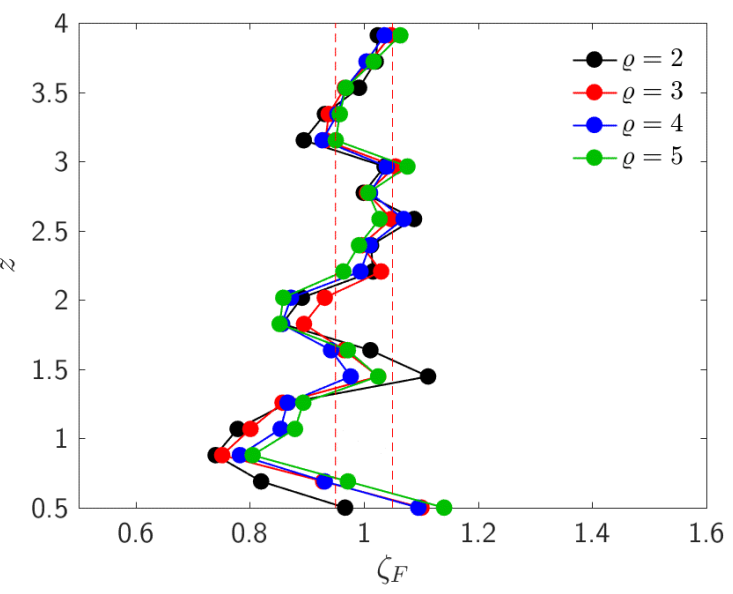

(d) $\phi=0.4$

Figure 12: $\zeta_{F}\left(z, \phi_{b}\right)$ as a function of the filter size $\varrho$ and the particle concentration $\phi_{b}$. Red dashed lines represent deviations of $\pm 5 \%$ from unity (corresponding to the bulk value). 


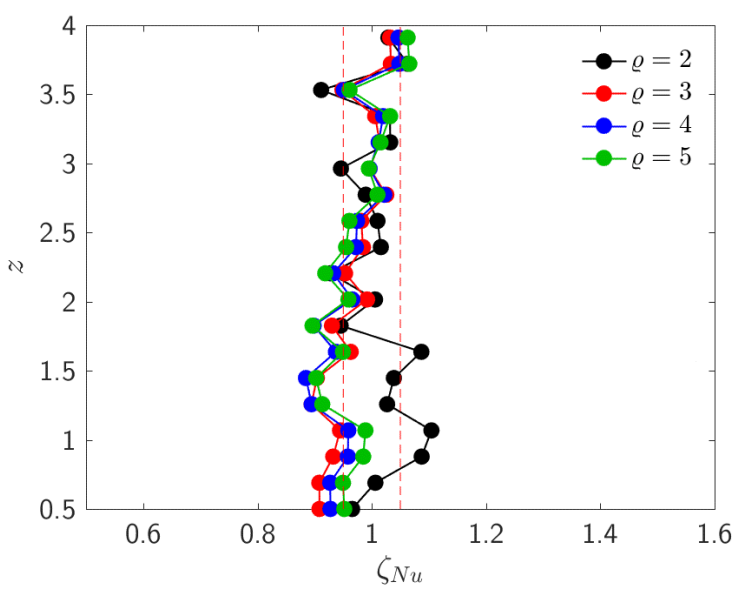

(a) $\phi=0.1$

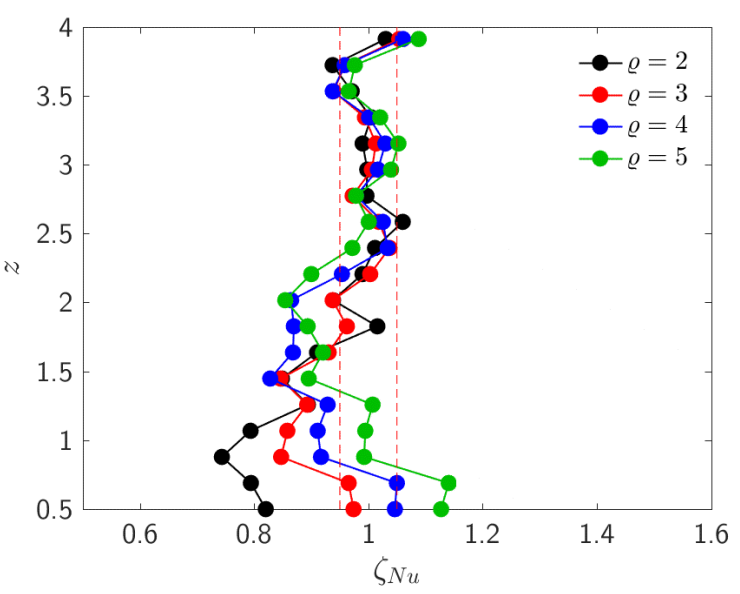

(c) $\phi=0.3$

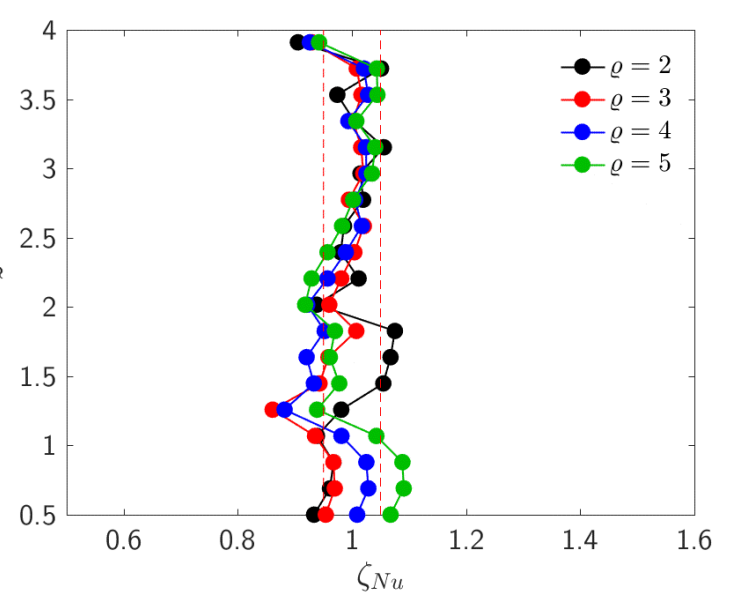

(b) $\phi=0.2$

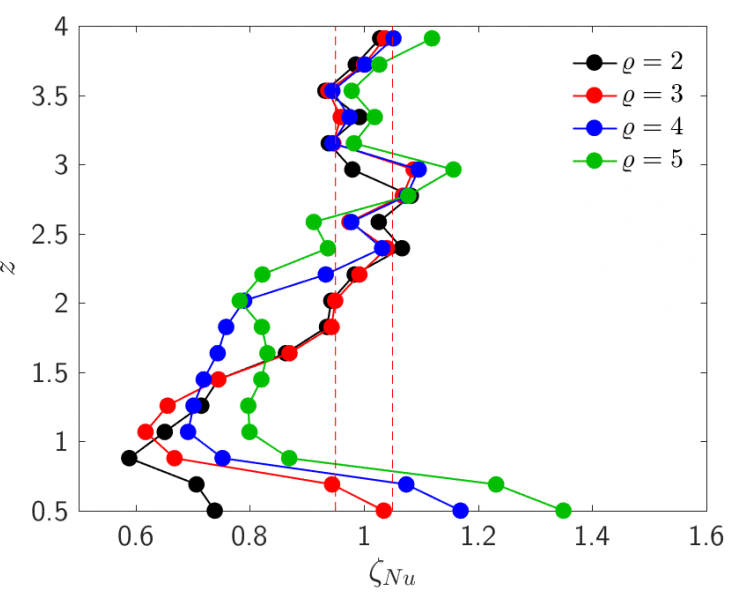

(d) $\phi=0.4$

Figure 13: $\zeta_{N u}\left(z, \phi_{b}\right)$ as a function of the filter size $\varrho$ and the particle concentration $\phi_{b}$. Red dashed lines represent deviations of $\pm 5 \%$ from unity (corresponding to the bulk value). 


\section{Summary}

We studied momentum, heat and mass transfer in dense gas-particle suspensions by means of Particle-Resolved Direct Numerical Simulation (PR-DNS) in wall bounded domains.

We found that the presence of walls induces a particle volume concentration field that is inhomogeneous in the wall-normal direction. We expressed this rather well-known observation as a series of perturbations with respect to an homogeneous bulk value. We employed particle simulations to obtain a correlation for the first term of our perturbation expansion. This now allows to accurately predict wall normal profiles of the particle volume concentration without performing expensive DEM-based simulations. Our correlation is valid in the range $0.05<\bar{\phi}<0.65$, and provides new insight on wall effects in dilute suspensions that are bounded by flat walls.

Next, we demonstrated that perturbations of the particle concentration fields have a significant effect on momentum, heat and mass transfer in the vicinity of walls. This is best illustrated by the peculiar wall normal profiles for the flow variables (i.e., velocity and temperature) documented in our present study. Most remarkably, we found that such profiles do not depend significantly on the Reynolds number when scaled with respect to their bulk value, i.e. the Reynolds number does not affect the shape of the profiles.

Such profiles allow to define a particle induced 'boundary layer' in which the fields differ from the bulk (homogeneous) value. In the present work, we proposed correlations to capture the most relevant physical phenomena happening in this particle induced boundary layer. Specifically, we obtained expressions for the scalar value at the wall, as well as the scalar gradient in the layer. Surprisingly, the latter is non zero despite we used adiabatic walls. Furthermore we provide expressions for (i) the maximum value of the velocity field in the layer, and (ii) the wall normal coordinate $z_{\max }$ at which this maximum occurs. These two quantities together with the observation that the velocity field is almost parabolic in $\left[0, z_{\max }\right]$ allows us to predict the wall shear stress exerted by the gas-particle system.

Finally we studied the interphase transfer coefficients for momentum (drag coefficient) and heat or mass transfer (i.e., the Nusselt or Sherwood number). Also in this case, we were able to obtain scaled wall-normal profiles that are independent on the Reynolds number. Unfortunately, we were not able to obtain a simple correlation for the wall correction functions of drag and heat or mass transfer. However, we tabulated values instead, which provide the basis for the correction of the Nusselt (or Sherwood) number, as well as the drag coefficient of particles situated at $0.5<z<2.5$ from the bounding wall. Most remarkably, for the Nusselt number an additional complexity arises, since the corrections are sensitive to the filter size $\rho$. This is due to the strong temperature gradient near the wall, which - in contrast to the strongly fluctuating mean velocity - causes a dependency of the filtered fluid temperature on the filter size.

This work was focussed on providing a fundamental understanding of wall-induced variations of momentum, heat and mass transfer coefficients in dense gas-particle suspensions. The development of a rigorous wall treatment (e.g., in the form of 'wall functions' to correct Nusselt numbers) would require further theoretical, experimental and most likely even more numerical studies. For example, the scaling we employed to remove the dependency on the Reynolds number may not be valid outside the range $100<R e<400$ investigated by us. A departure from such a 'Reynolds number invariant' regime would increase the dimensionality of the expression needed to correct for wall effects (i.e., it would require to explicitly account for the Reynolds number). One might speculate that such a departue would be most noticeable in the turbulent flow regime, since turbulent agitation would most likely smoothen out the profiles of interest. Thus, exploring wall effects at Reynolds numbers beyond 400 would be a logical next step. Furthermore, we did not provide an expression for wall corrections to the interphase transfer coefficients. This limitation was imposed mainly by the lack of a deeper physical understanding (in the context of a theoretical model) that causes these corrections. Thus, the development of a simple theoretical model to explain the observed variability in the transfer coefficients appears to offer a fruitful direction for future research.

\section{Acknowledgement}

The authors acknowledge support by the European Commission through FP7 Grant agreement 604656 (NanoSim), and the NAWI Graz project by providing access to dcluster.tugraz.at. CFDEM is a registered trademark of DCS Computing $\mathrm{GmbH}$. The computational results presented have been achieved (in part) using the Vienna Scientific Cluster (VSC-3). 


\section{Appendix A. Additional data}

Appendix A.1. Tabulated values for Drag and Nusselt wall corrections

Table A.3: Values of $\zeta_{F}\left(z, \phi_{b}\right)$ shown in figure 12a and figure 12b.

\begin{tabular}{|c|c|c|c|c|c|c|c|c|}
\hline \multirow[t]{2}{*}{$z$} & \multicolumn{4}{|c|}{$\phi_{b}=0.1$} & \multicolumn{4}{|c|}{$\phi_{b}=0.2$} \\
\hline & $\varrho=2$ & $\varrho=3$ & $\varrho=4$ & $\varrho=5$ & $\varrho=2$ & $\varrho=3$ & $\varrho=4$ & $\varrho=5$ \\
\hline 0.500 & 1.270 & 1.184 & 1.181 & 1.177 & 1.285 & 1.200 & 1.150 & 1.104 \\
\hline 0.689 & 1.155 & 1.117 & 1.129 & 1.130 & 1.190 & 1.077 & 1.030 & 0.992 \\
\hline 0.879 & 1.108 & 1.057 & 1.076 & 1.078 & 1.158 & 1.090 & 1.051 & 1.014 \\
\hline 1.068 & 0.988 & 1.033 & 1.044 & 1.051 & 1.057 & 1.096 & 1.050 & 1.025 \\
\hline 1.258 & 0.820 & 0.891 & 0.887 & 0.905 & 0.949 & 1.056 & 1.027 & 0.989 \\
\hline 1.447 & 1.054 & 1.038 & 1.024 & 1.038 & 1.027 & 1.061 & 1.046 & 1.012 \\
\hline 1.637 & 1.111 & 1.050 & 1.072 & 1.070 & 1.098 & 1.080 & 1.126 & 1.094 \\
\hline 1.826 & 1.064 & 1.021 & 1.050 & 1.033 & 1.004 & 0.973 & 1.032 & 1.000 \\
\hline 2.016 & 1.003 & 0.993 & 0.993 & 0.995 & 1.051 & 1.033 & 1.063 & 1.076 \\
\hline 2.205 & 0.958 & 0.956 & 0.950 & 0.980 & 0.952 & 0.967 & 0.958 & 0.993 \\
\hline 2.395 & 1.000 & 0.999 & 1.005 & 1.023 & 1.030 & 1.011 & 1.011 & 1.039 \\
\hline 2.584 & 1.025 & 0.997 & 0.998 & 0.970 & 1.004 & 0.988 & 0.980 & 0.984 \\
\hline 2.774 & 1.016 & 1.016 & 1.032 & 1.016 & 1.018 & 1.030 & 1.032 & 1.014 \\
\hline 2.963 & 1.039 & 1.024 & 1.025 & 1.026 & 1.027 & 0.999 & 0.994 & 0.980 \\
\hline 3.153 & 1.072 & 1.084 & 1.083 & 1.097 & 0.989 & 1.007 & 0.997 & 0.983 \\
\hline 3.342 & 0.983 & 1.024 & 1.009 & 1.000 & 0.992 & 0.999 & 1.003 & 0.999 \\
\hline 3.532 & 0.967 & 0.978 & 0.982 & 0.984 & 0.922 & 0.927 & 0.924 & 0.920 \\
\hline 3.721 & 0.977 & 0.986 & 0.989 & 0.986 & 1.033 & 1.034 & 1.045 & 1.039 \\
\hline 3.911 & 0.963 & 0.942 & 0.928 & 0.930 & 1.001 & 1.008 & 1.020 & 1.011 \\
\hline
\end{tabular}


Table A.4: Values of $\zeta_{F}\left(z, \phi_{b}\right)$ shown in figure $12 \mathrm{c}$ and figure $12 \mathrm{~d}$.

\begin{tabular}{|c|c|c|c|c|c|c|c|c|}
\hline \multirow[t]{2}{*}{$z$} & \multicolumn{4}{|c|}{$\phi_{b}=0.3$} & \multicolumn{4}{|c|}{$\phi_{b}=0.4$} \\
\hline & $\varrho=2$ & $\varrho=3$ & $\varrho=4$ & $\varrho=5$ & $\varrho=2$ & $\varrho=3$ & $\varrho=4$ & $\varrho=5$ \\
\hline 0.500 & 1.111 & 1.157 & 1.159 & 1.180 & 0.967 & 1.101 & 1.095 & 1.140 \\
\hline 0.689 & 0.945 & 0.976 & 1.003 & 1.017 & 0.820 & 0.928 & 0.931 & 0.972 \\
\hline 0.879 & 0.901 & 0.858 & 0.880 & 0.889 & 0.740 & 0.752 & 0.783 & 0.805 \\
\hline 1.068 & 0.777 & 0.827 & 0.847 & 0.868 & 0.779 & 0.801 & 0.854 & 0.879 \\
\hline 1.258 & 0.937 & 0.934 & 0.942 & 0.969 & 0.867 & 0.857 & 0.866 & 0.894 \\
\hline 1.447 & 1.023 & 0.991 & 0.965 & 0.996 & 1.112 & 1.025 & 0.977 & 1.025 \\
\hline 1.637 & 1.014 & 0.965 & 0.966 & 0.981 & 1.011 & 0.966 & 0.942 & 0.972 \\
\hline 1.826 & 0.920 & 0.927 & 0.924 & 0.917 & 0.857 & 0.895 & 0.856 & 0.852 \\
\hline 2.016 & 0.990 & 1.009 & 0.983 & 0.979 & 0.891 & 0.931 & 0.872 & 0.859 \\
\hline 2.205 & 0.970 & 0.965 & 0.935 & 0.940 & 1.016 & 1.030 & 0.994 & 0.964 \\
\hline 2.395 & 1.020 & 1.033 & 1.046 & 1.046 & 1.012 & 0.996 & 1.010 & 0.991 \\
\hline 2.584 & 1.068 & 1.040 & 1.059 & 1.036 & 1.088 & 1.048 & 1.069 & 1.027 \\
\hline 2.774 & 1.034 & 1.031 & 1.038 & 1.028 & 1.000 & 1.005 & 1.011 & 1.008 \\
\hline 2.963 & 0.997 & 1.017 & 1.020 & 1.038 & 1.036 & 1.055 & 1.040 & 1.076 \\
\hline 3.153 & 0.952 & 0.985 & 0.983 & 0.995 & 0.895 & 0.933 & 0.927 & 0.951 \\
\hline 3.342 & 0.963 & 0.963 & 0.962 & 0.961 & 0.931 & 0.938 & 0.953 & 0.958 \\
\hline 3.532 & 0.947 & 0.937 & 0.940 & 0.935 & 0.991 & 0.966 & 0.968 & 0.968 \\
\hline 3.721 & 1.007 & 0.995 & 0.989 & 0.987 & 1.021 & 1.011 & 1.004 & 1.017 \\
\hline 3.911 & 1.029 & 1.029 & 1.024 & 1.032 & 1.024 & 1.047 & 1.036 & 1.064 \\
\hline
\end{tabular}

Table A.5: Values of $\zeta_{N u}\left(z, \phi_{b}\right)$ shown in figure 13a and figure $13 \mathrm{~b}$.

\begin{tabular}{|c|c|c|c|c|c|c|c|c|}
\hline \multirow[t]{2}{*}{$z$} & \multicolumn{4}{|c|}{$\phi_{b}=0.1$} & \multicolumn{4}{|c|}{$\phi_{b}=0.2$} \\
\hline & $\varrho=2$ & $\varrho=3$ & $\varrho=4$ & $\varrho=5$ & $\varrho=2$ & $\varrho=3$ & $\varrho=4$ & $\varrho=5$ \\
\hline 0.500 & 0.966 & 0.908 & 0.928 & 0.952 & 0.934 & 0.954 & 1.009 & 1.068 \\
\hline 0.689 & 1.006 & 0.908 & 0.927 & 0.950 & 0.961 & 0.970 & 1.029 & 1.091 \\
\hline 0.879 & 1.087 & 0.933 & 0.958 & 0.985 & 0.968 & 0.967 & 1.025 & 1.088 \\
\hline 1.068 & 1.104 & 0.944 & 0.959 & 0.989 & 0.939 & 0.935 & 0.982 & 1.043 \\
\hline 1.258 & 1.027 & 0.894 & 0.895 & 0.913 & 0.981 & 0.861 & 0.882 & 0.939 \\
\hline 1.447 & 1.039 & 0.904 & 0.885 & 0.903 & 1.055 & 0.943 & 0.933 & 0.978 \\
\hline 1.637 & 1.087 & 0.963 & 0.937 & 0.950 & 1.068 & 0.959 & 0.921 & 0.961 \\
\hline 1.826 & 0.945 & 0.930 & 0.898 & 0.896 & 1.075 & 1.008 & 0.952 & 0.970 \\
\hline 2.016 & 1.006 & 0.992 & 0.966 & 0.959 & 0.938 & 0.960 & 0.922 & 0.918 \\
\hline 2.205 & 0.927 & 0.953 & 0.932 & 0.919 & 1.012 & 0.981 & 0.957 & 0.929 \\
\hline 2.395 & 1.016 & 0.985 & 0.972 & 0.955 & 0.980 & 1.004 & 0.989 & 0.957 \\
\hline 2.584 & 1.010 & 0.982 & 0.974 & 0.961 & 0.986 & 1.021 & 1.017 & 0.983 \\
\hline 2.774 & 0.989 & 1.025 & 1.022 & 1.010 & 1.019 & 0.995 & 1.006 & 1.002 \\
\hline 2.963 & 0.946 & 0.996 & 0.996 & 0.995 & 1.015 & 1.020 & 1.025 & 1.035 \\
\hline 3.153 & 1.032 & 1.014 & 1.012 & 1.015 & 1.056 & 1.016 & 1.025 & 1.041 \\
\hline 3.342 & 1.032 & 1.006 & 1.020 & 1.032 & 1.004 & 1.002 & 0.994 & 1.008 \\
\hline 3.532 & 0.911 & 0.949 & 0.951 & 0.961 & 0.974 & 1.017 & 1.028 & 1.045 \\
\hline 3.721 & 1.063 & 1.033 & 1.048 & 1.066 & 1.051 & 1.008 & 1.021 & 1.044 \\
\hline 3.911 & 1.029 & 1.032 & 1.047 & 1.063 & 0.905 & 0.930 & 0.926 & 0.942 \\
\hline
\end{tabular}


Table A.6: Values of $\zeta_{N u}\left(z, \phi_{b}\right)$ shown in figure $13 \mathrm{c}$ and figure $13 \mathrm{~d}$.

\begin{tabular}{|c|c|c|c|c|c|c|c|c|}
\hline \multirow[t]{2}{*}{$z$} & \multicolumn{4}{|c|}{$\phi_{b}=0.3$} & \multicolumn{4}{|c|}{$\phi_{b}=0.4$} \\
\hline & $\varrho=2$ & $\varrho=3$ & $\varrho=4$ & $\varrho=5$ & $\varrho=2$ & $\varrho=3$ & $\varrho=4$ & $\varrho=5$ \\
\hline 0.500 & 0.820 & 0.974 & 1.046 & 1.127 & 0.738 & 1.035 & 1.169 & 1.350 \\
\hline 0.689 & 0.794 & 0.965 & 1.050 & 1.141 & 0.706 & 0.944 & 1.074 & 1.232 \\
\hline 0.879 & 0.744 & 0.847 & 0.917 & 0.993 & 0.589 & 0.668 & 0.752 & 0.869 \\
\hline 1.068 & 0.794 & 0.858 & 0.911 & 0.995 & 0.651 & 0.617 & 0.692 & 0.799 \\
\hline 1.258 & 0.894 & 0.893 & 0.929 & 1.007 & 0.715 & 0.656 & 0.701 & 0.797 \\
\hline 1.447 & 0.849 & 0.846 & 0.828 & 0.896 & 0.745 & 0.745 & 0.719 & 0.820 \\
\hline 1.637 & 0.910 & 0.930 & 0.868 & 0.920 & 0.863 & 0.869 & 0.744 & 0.831 \\
\hline 1.826 & 1.016 & 0.962 & 0.869 & 0.894 & 0.935 & 0.943 & 0.759 & 0.821 \\
\hline 2.016 & 0.938 & 0.937 & 0.865 & 0.854 & 0.943 & 0.950 & 0.790 & 0.782 \\
\hline 2.205 & 0.990 & 1.003 & 0.954 & 0.900 & 0.984 & 0.992 & 0.933 & 0.822 \\
\hline 2.395 & 1.012 & 1.037 & 1.034 & 0.972 & 1.066 & 1.040 & 1.032 & 0.937 \\
\hline 2.584 & 1.060 & 1.018 & 1.025 & 1.001 & 1.026 & 0.975 & 0.978 & 0.912 \\
\hline 2.774 & 0.997 & 0.972 & 0.978 & 0.978 & 1.082 & 1.068 & 1.075 & 1.078 \\
\hline 2.963 & 0.998 & 1.006 & 1.017 & 1.040 & 0.980 & 1.087 & 1.096 & 1.157 \\
\hline 3.153 & 0.990 & 1.013 & 1.030 & 1.053 & 0.938 & 0.946 & 0.945 & 0.982 \\
\hline 3.342 & 1.004 & 0.994 & 1.001 & 1.021 & 0.992 & 0.959 & 0.975 & 1.019 \\
\hline 3.532 & 0.971 & 0.939 & 0.938 & 0.966 & 0.932 & 0.937 & 0.945 & 0.978 \\
\hline 3.721 & 0.937 & 0.959 & 0.958 & 0.976 & 0.986 & 1.000 & 1.001 & 1.027 \\
\hline 3.911 & 1.030 & 1.054 & 1.060 & 1.088 & 1.028 & 1.037 & 1.052 & 1.119 \\
\hline
\end{tabular}

\section{Appendix A.2. Drag and Nusselt profiles for different Reynolds numbers}

In this section we show the scaled profiles for drag and Nusselt number at different Reynolds numbers. The peculiar characteristics are visible for different values of $\phi_{p}$ rather than for different Reynolds numbers. 


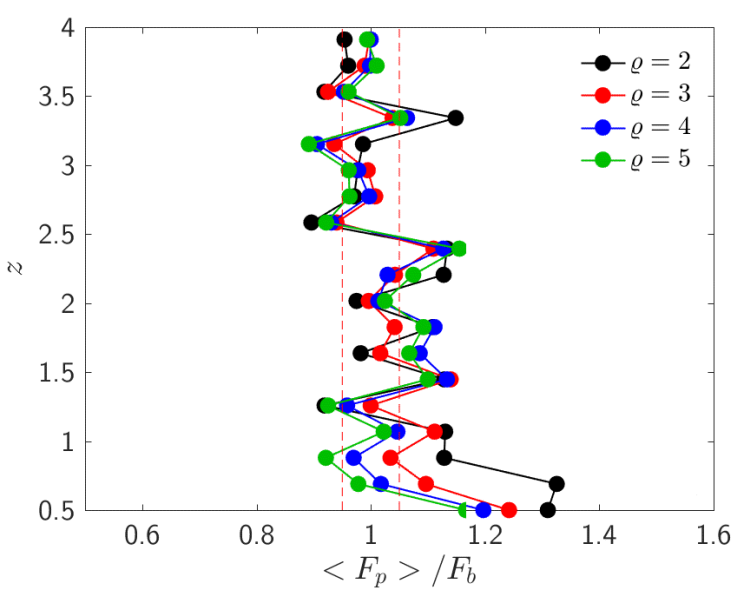

(a) $\phi=0.1$

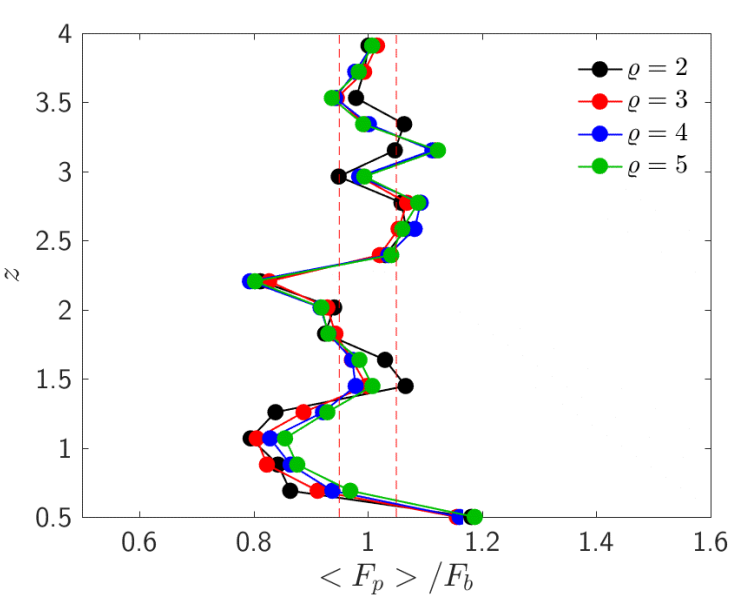

(c) $\phi=0.3$

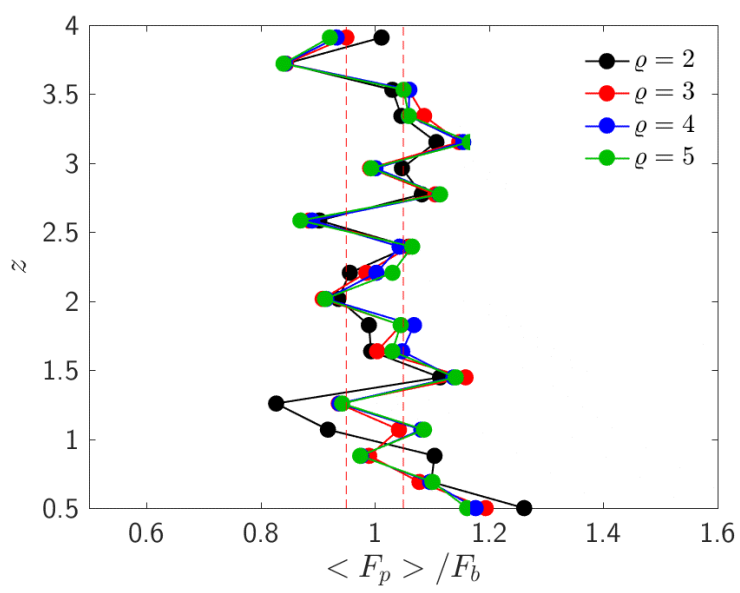

(b) $\phi=0.2$

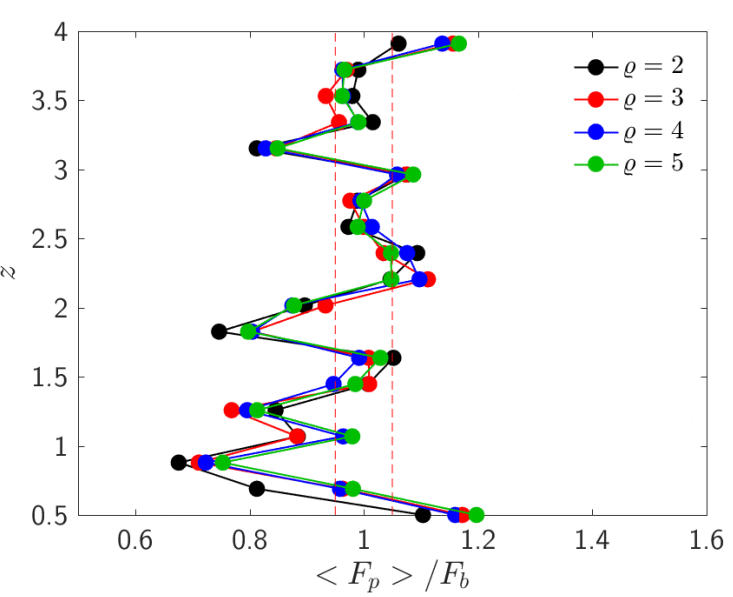

(d) $\phi=0.4$

Figure A.14: Scaled drag for $R e=100$. 


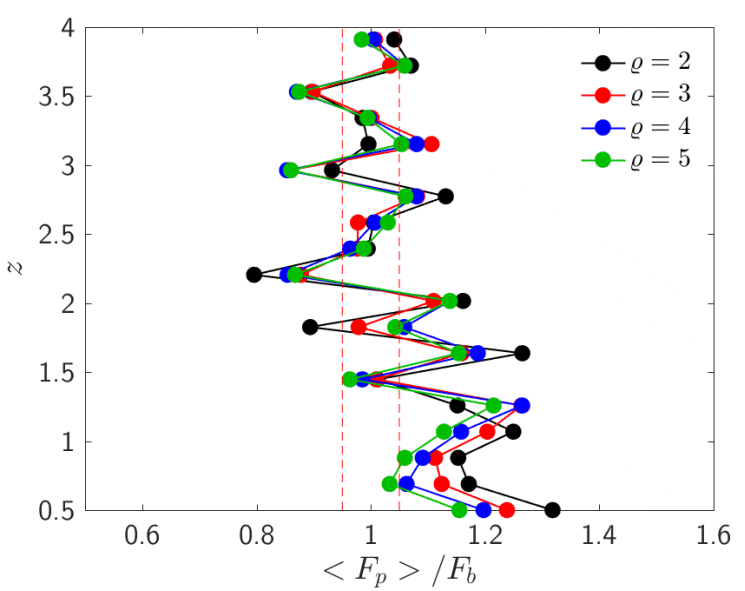

(a) $\phi=0.1$

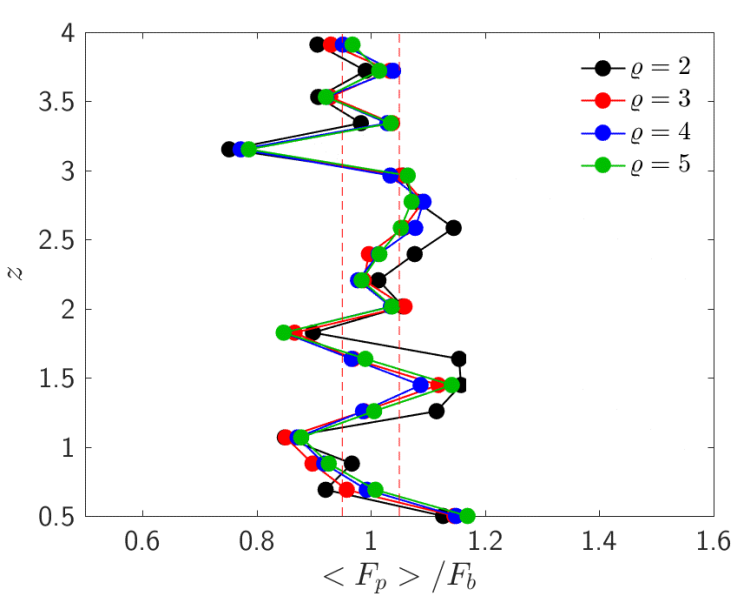

(c) $\phi=0.3$

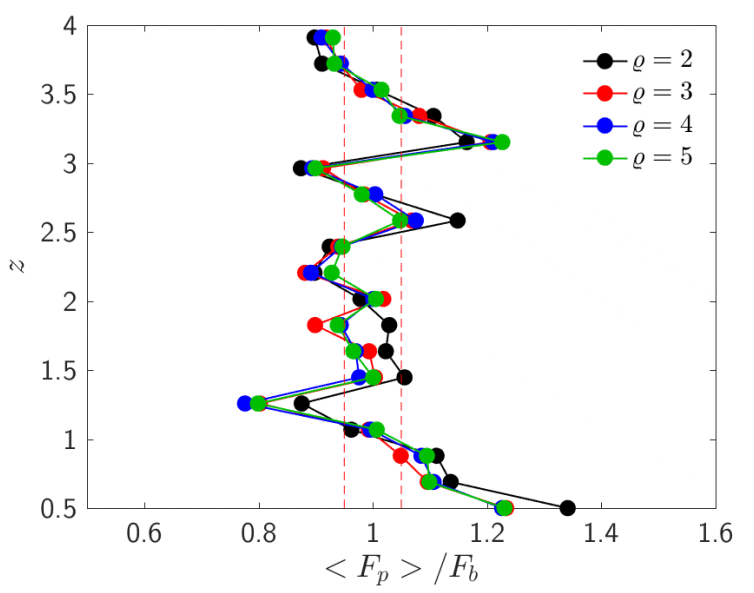

(b) $\phi=0.2$

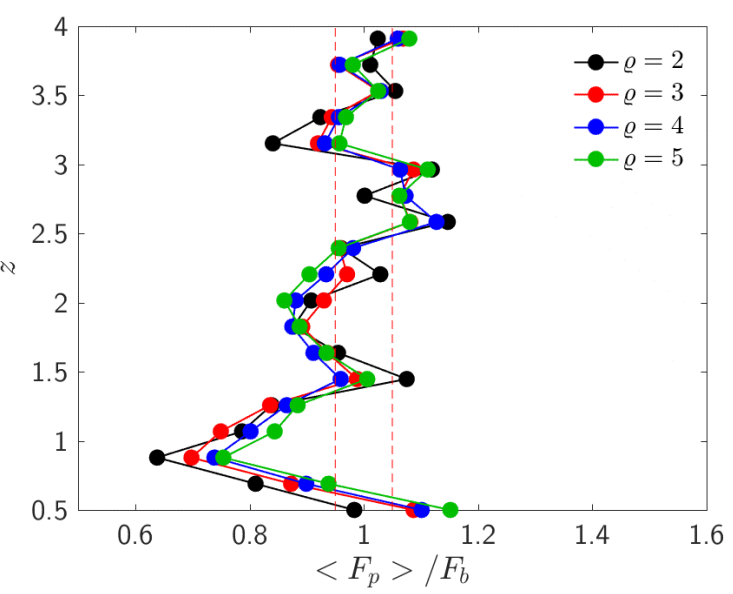

(d) $\phi=0.4$

Figure A.15: Scaled drag for $R e=200$. 


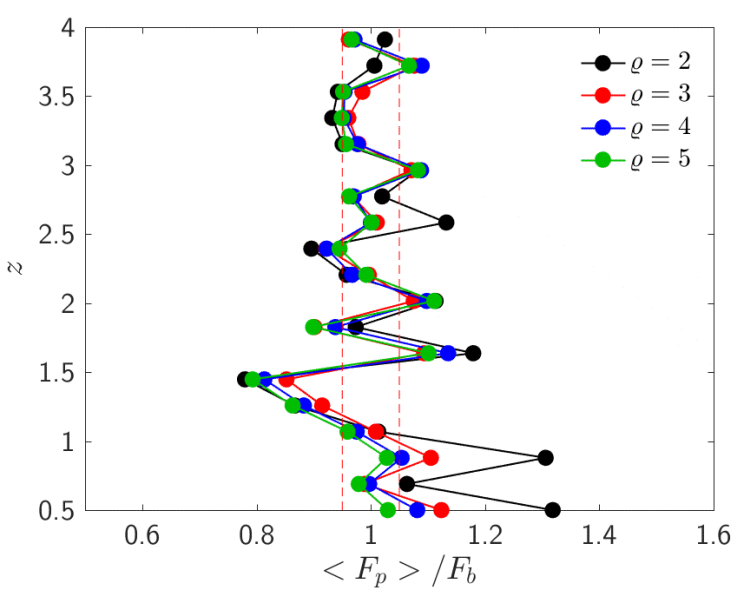

(a) $\phi=0.1$

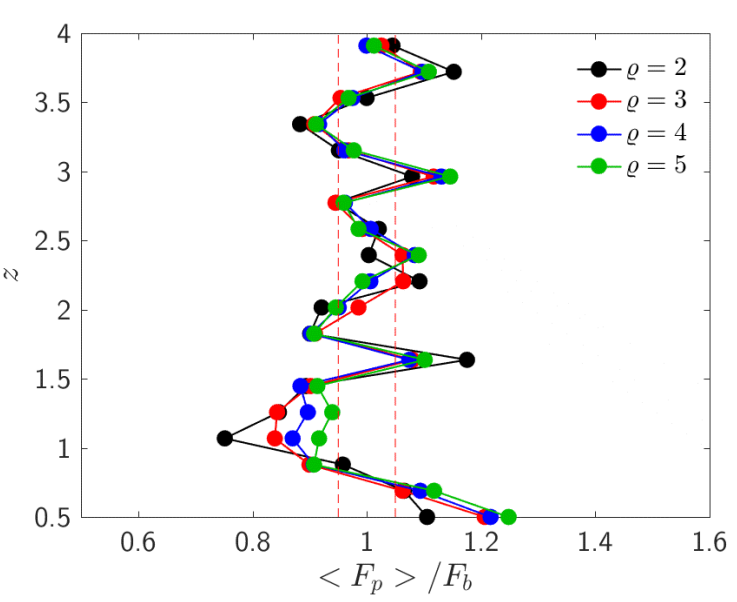

(c) $\phi=0.3$

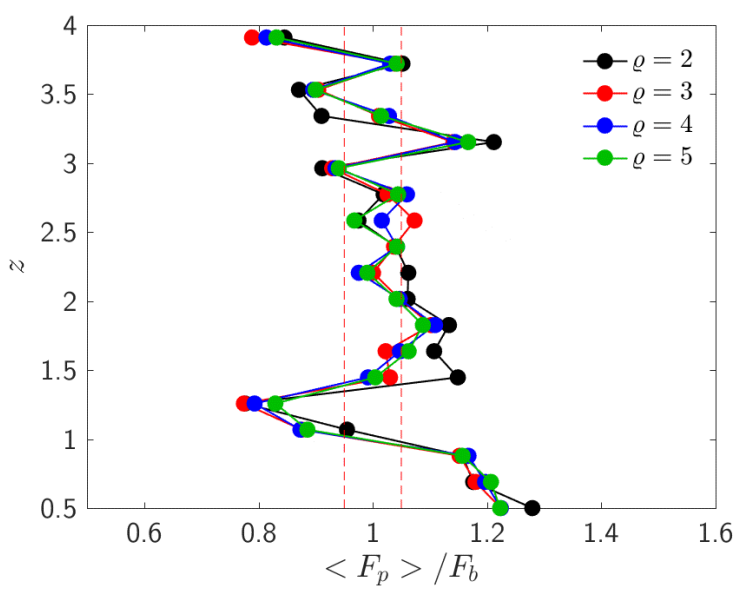

(b) $\phi=0.2$

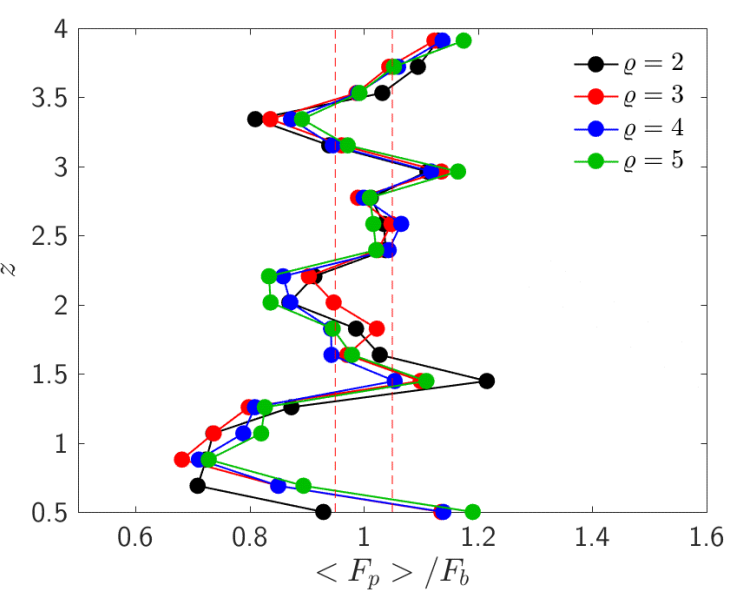

(d) $\phi=0.4$

Figure A.16: Scaled drag for $R e=300$. 


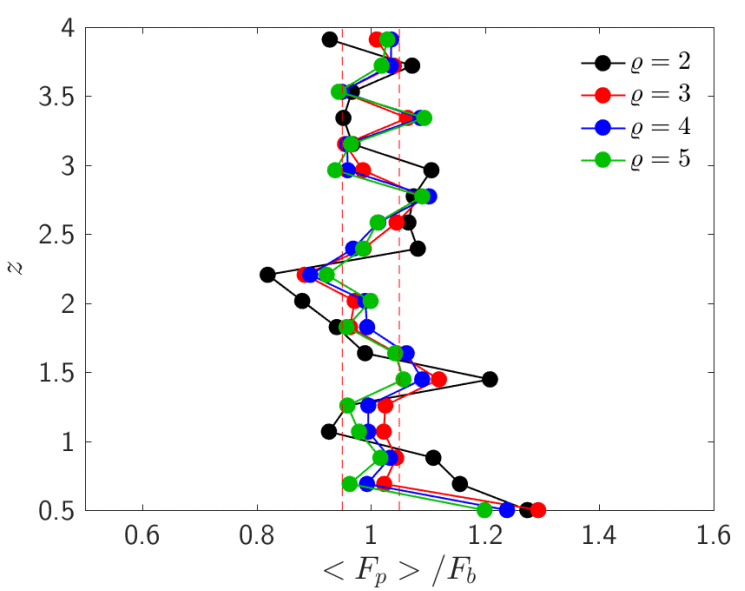

(a) $\phi=0.1$

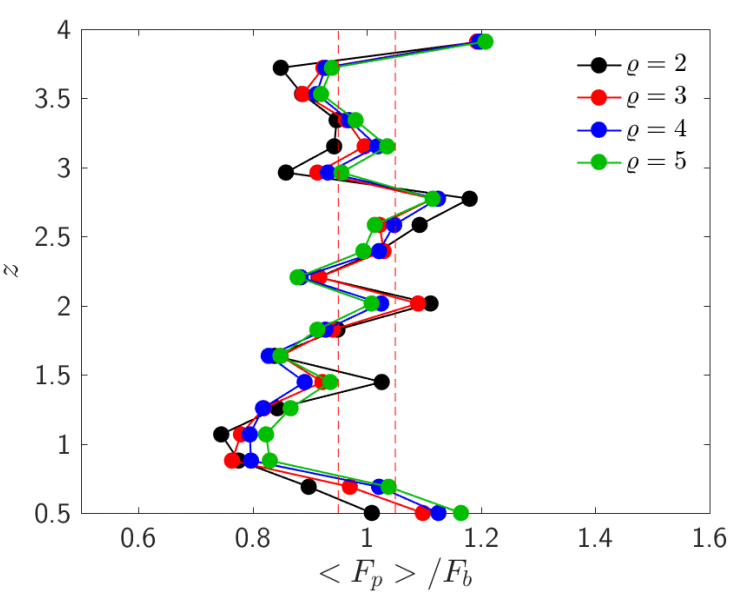

(c) $\phi=0.3$

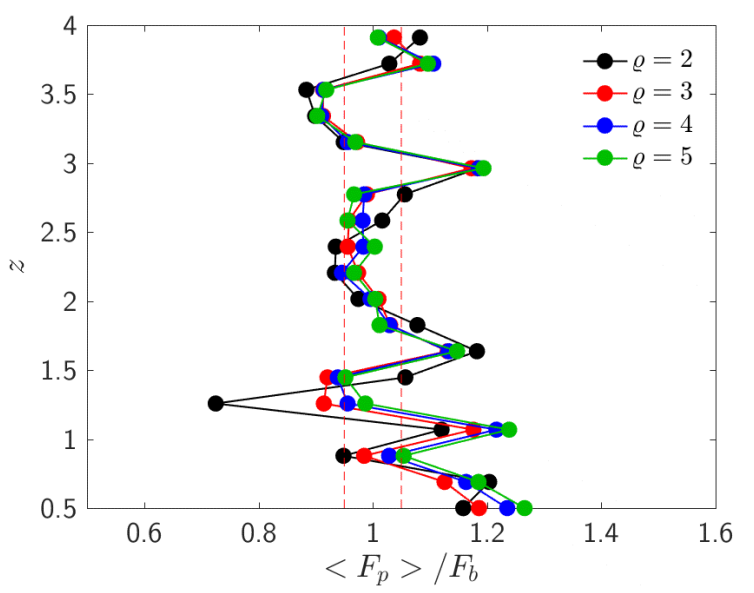

(b) $\phi=0.2$

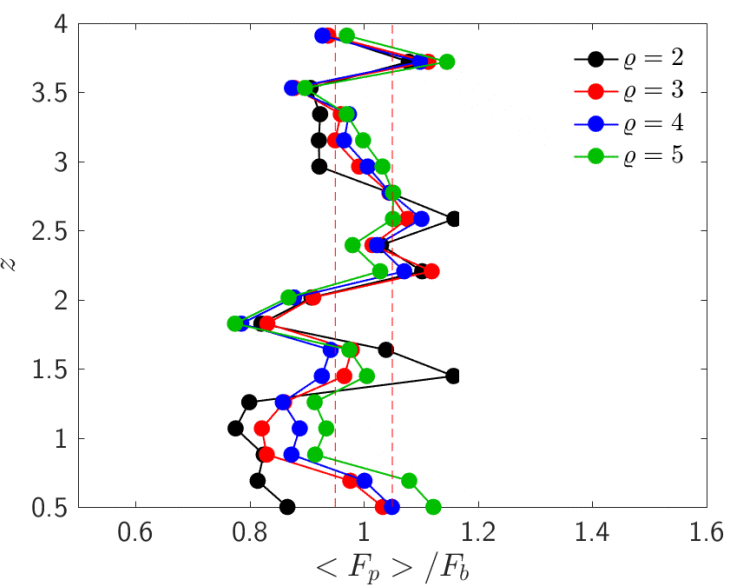

(d) $\phi=0.4$

Figure A.17: Scaled drag for $R e=400$. 


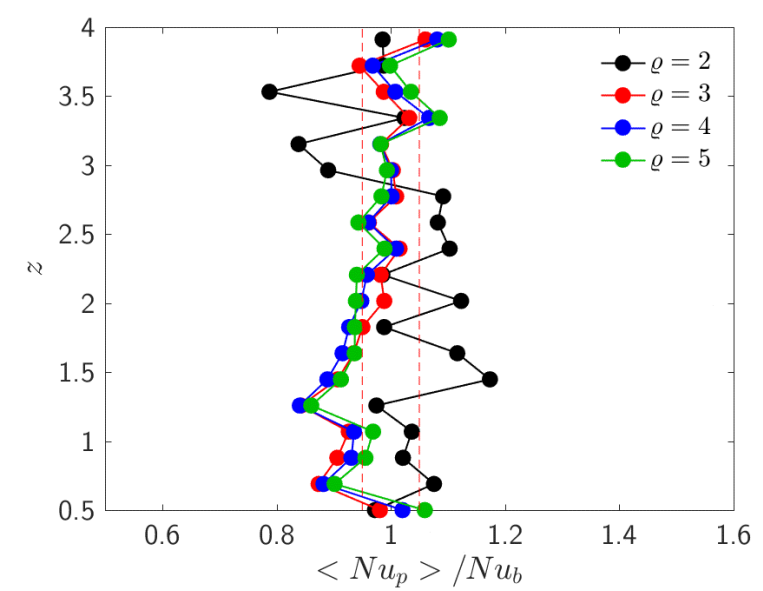

(a) $\phi=0.1$

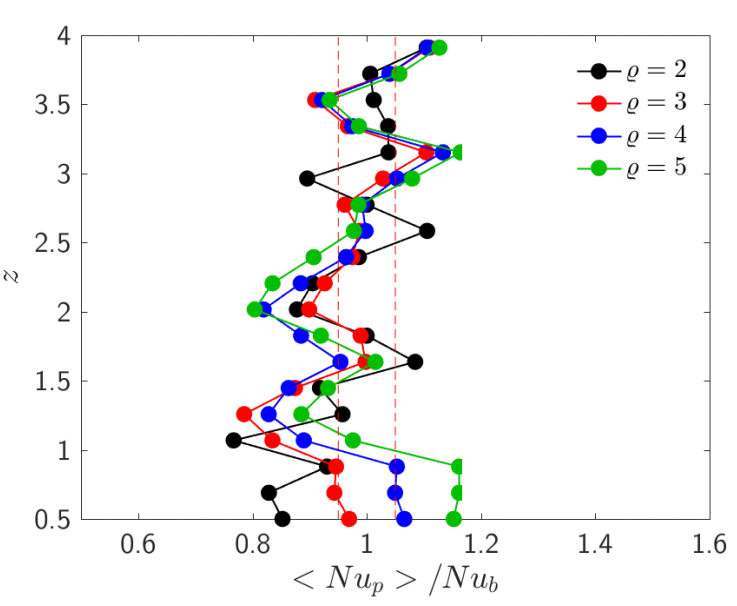

(c) $\phi=0.3$

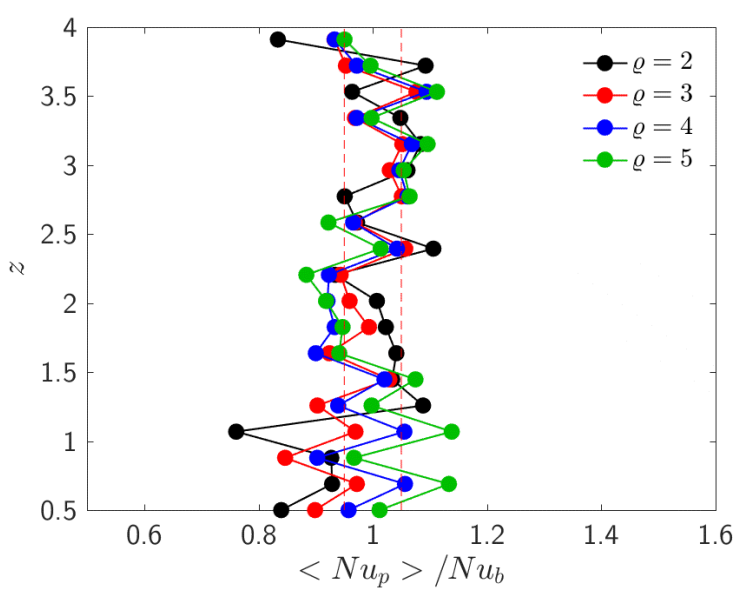

(b) $\phi=0.2$

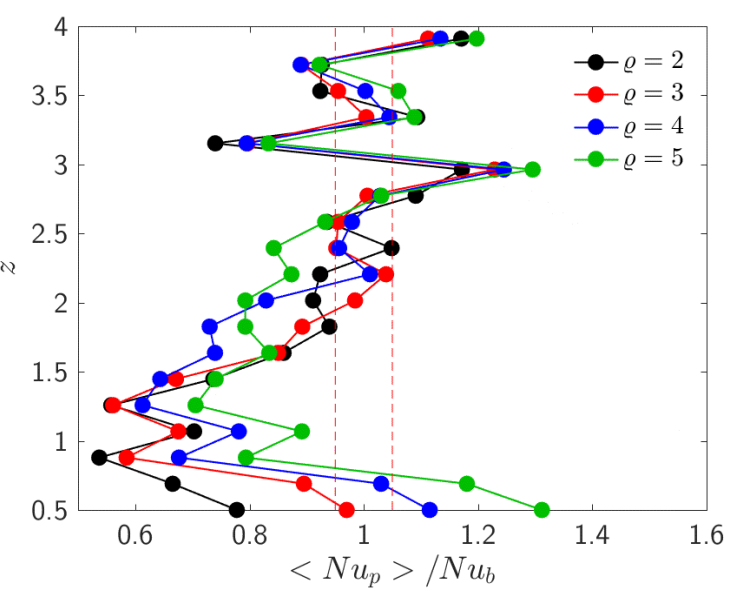

(d) $\phi=0.4$

Figure A.18: Scaled Nusslet for $R e=100$. 


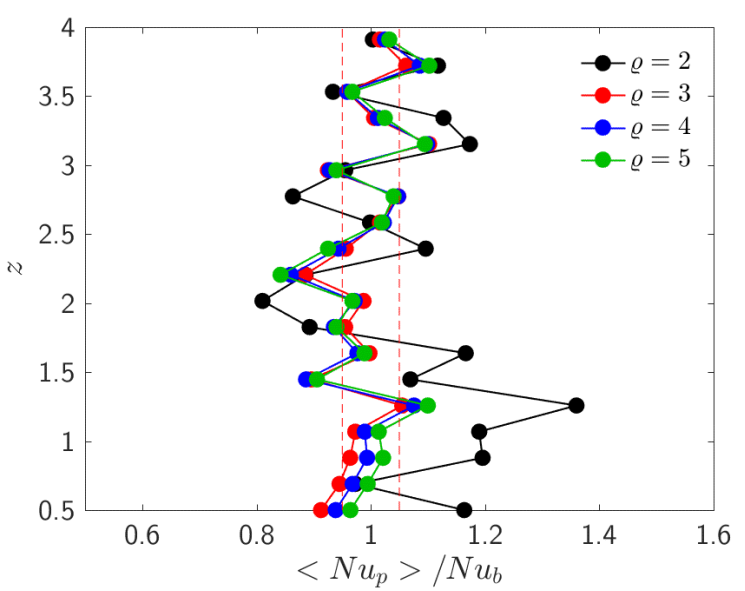

(a) $\phi=0.1$

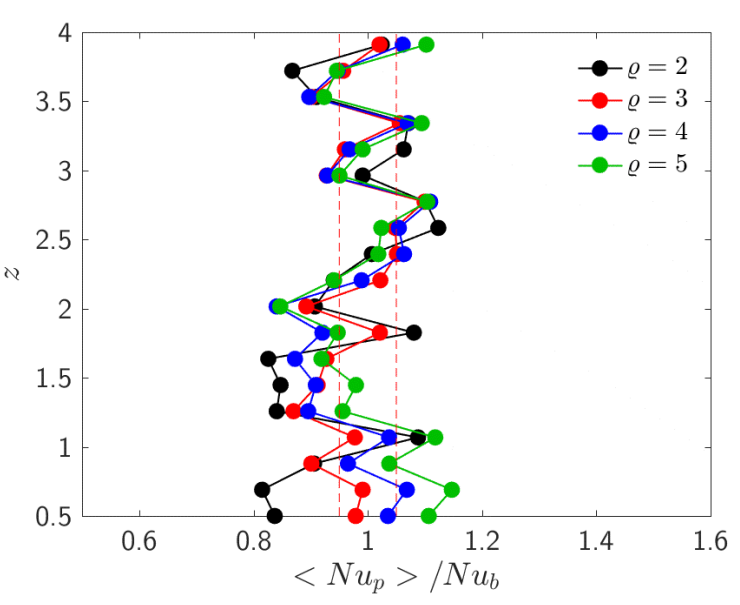

(c) $\phi=0.3$

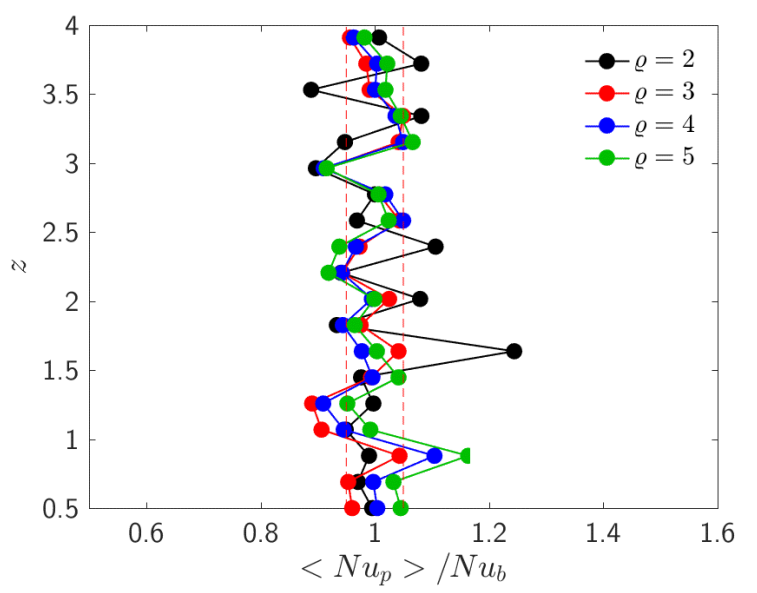

(b) $\phi=0.2$

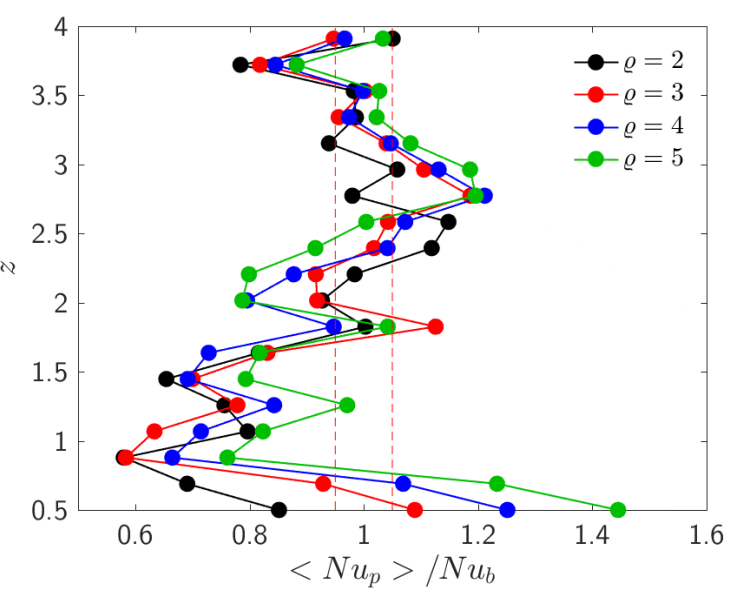

(d) $\phi=0.4$

Figure A.19: Scaled Nusslet for $R e=200$. 


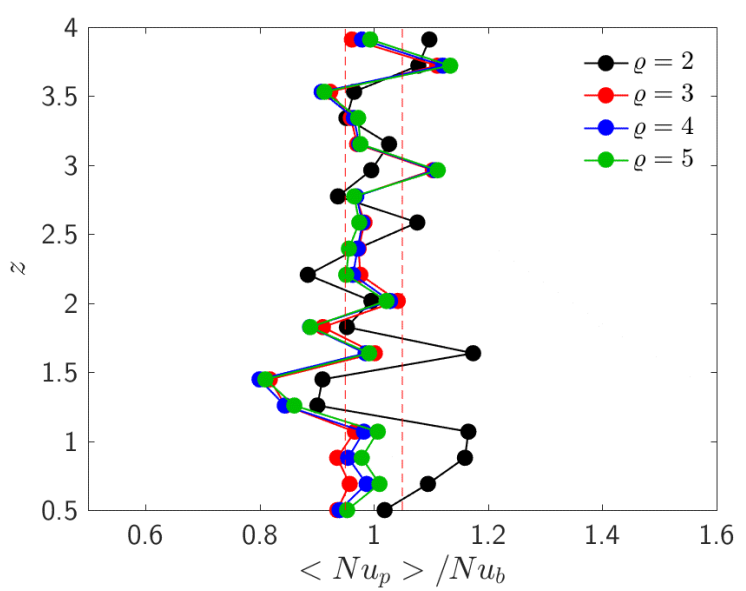

(a) $\phi=0.1$

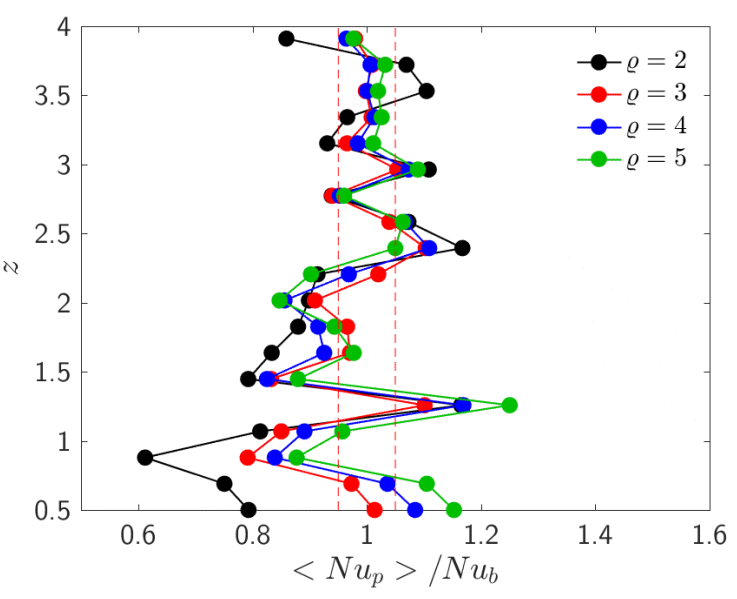

(c) $\phi=0.3$

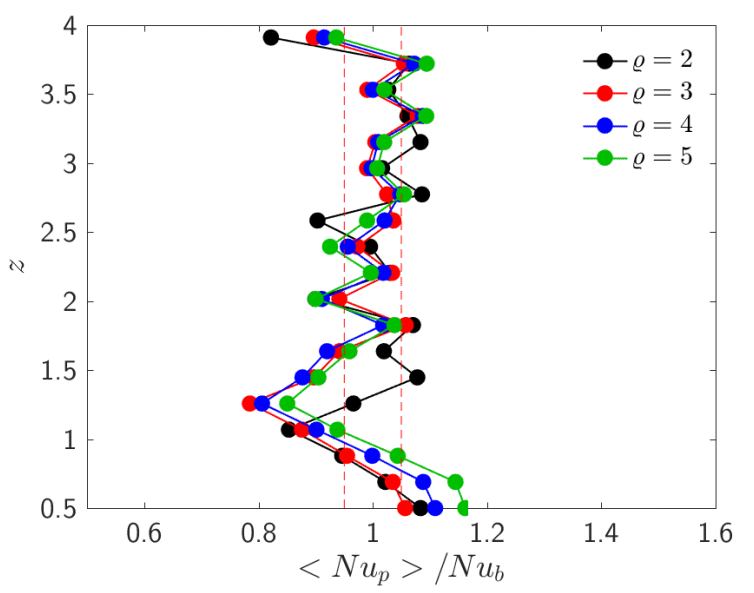

(b) $\phi=0.2$

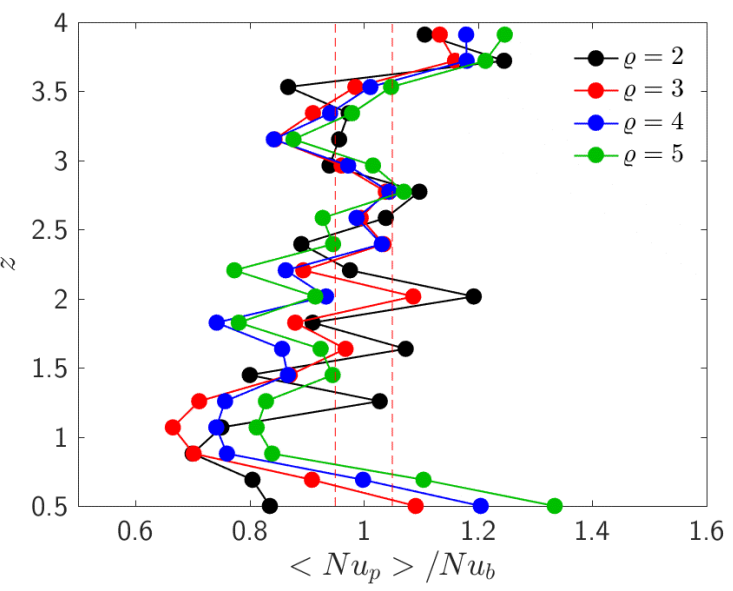

(d) $\phi=0.4$

Figure A.20: Scaled Nusslet for $R e=300$. 


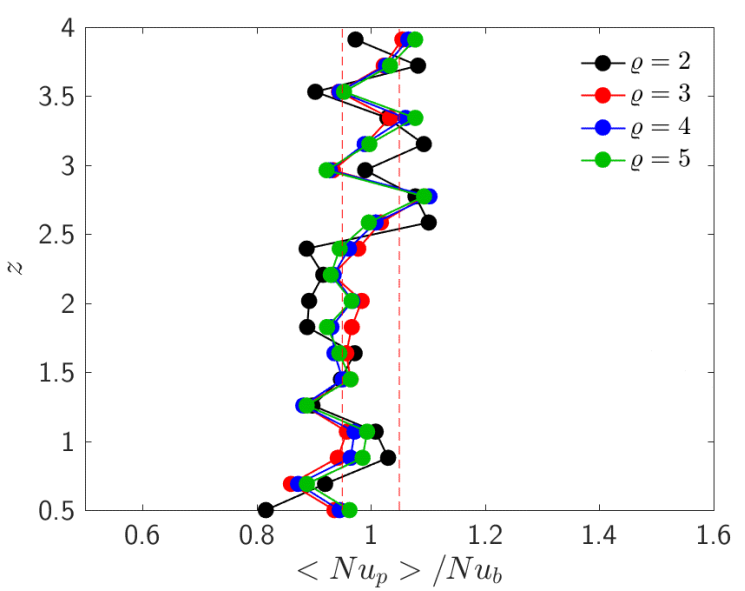

(a) $\phi=0.1$

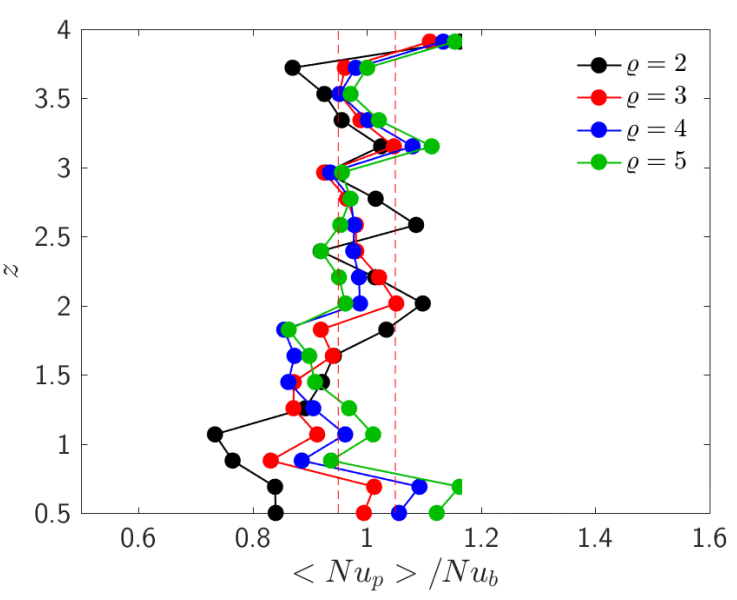

(c) $\phi=0.3$

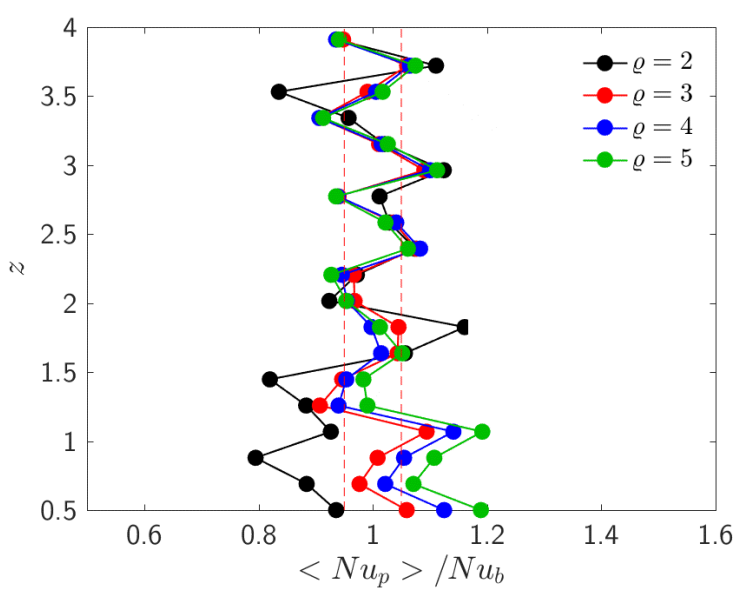

(b) $\phi=0.2$

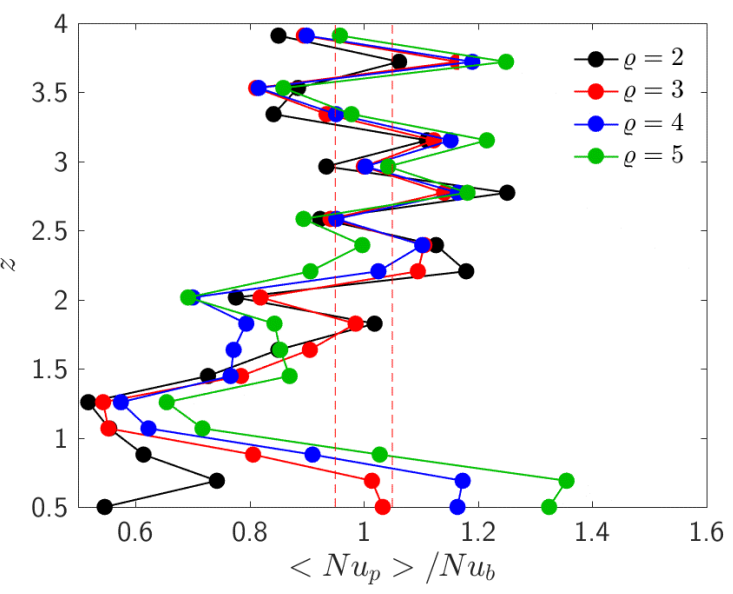

(d) $\phi=0.4$

Figure A.21: Scaled Nusslet for $R e=400$. 
[1] B. Gutmann, M. Köckinger, G. Glotz, T. Ciaglia, E. Slama, M. Zadravec, S. Pfanner, M. C. Maier, H. Gruber-Wölfler, C. Oliver Kappe, Design and 3D printing of a stainless steel reactor for continuous difluoromethylations using fluoroform, Reaction Chemistry \& Engineering 2 (6) (2017) 919-927. doi:10.1039/C7RE00176B.

URL http://xlink.rsc.org/?DOI=C7RE00176B

[2] A. G. Dixon, Local transport and reaction rates in a fixed bed reactor tube: Endothermic steam methane reforming, Chemical Engineering Science 168 (2017) 156-177. doi:10.1016/j.ces.2017.04.039.

URL http://linkinghub.elsevier.com/retrieve/pii/S0009250917302877

[3] Y. Dong, B. Sosna, O. Korup, F. Rosowski, R. Horn, Investigation of radial heat transfer in a fixed-bed reactor: CFD simulations and profile measurements, Chemical Engineering Journal 317 (2017) 204-214. doi:10.1016/j.cej.2017.02.063.

URL http://dx.doi.org/10.1016/j.cej.2017.02.063 http://linkinghub.elsevier.com/retrieve/pii/S1385894717302279

[4] G. Zanganeh, A. Pedretti, S. Zavattoni, M. Barbato, A. Steinfeld, Packed-bed thermal storage for concentrated solar power Pilot-scale demonstration and industrial-scale design, Solar Energy 86 (10) (2012) 3084-3098. doi:10.1016/j.solener.2012.07.019. URL http://dx.doi.org/10.1016/j.solener.2012.07.019 http://linkinghub.elsevier.com/retrieve/pii/S0038092X12002812

[5] J. Marti, A. Haselbacher, A. Steinfeld, A numerical investigation of gas-particle suspensions as heat transfer media for high-temperature concentrated solar power, International Journal of Heat and Mass Transfer 90 (2015) 1056-1070. doi:10.1016/j.ijheatmasstransfer.2015.07.033. URL http://dx.doi.org/10.1016/j.ijheatmasstransfer.2015.07.033

[6] J. J. Derksen, Simulations of granular bed erosion due to laminar shear flow near the critical Shields number, Physics of Fluids 23 (11) (2011) 113303. doi:10.1063/1.3660258.

URL http://scitation.aip.org/content/aip/journal/pof2/23/11/10.1063/1.3660258

[7] J. J. DERKSEN, R. A. LARSEN, Drag and lift forces on random assemblies of wall-attached spheres in low-Reynolds-number shear flow, Journal of Fluid Mechanics 673 (2011) (2011) 548-573. doi:10.1017/S0022112010006403. URL http: //www . journals . cambridge.org/abstract_S0022112010006403

[8] J. Derksen, G. Reynolds, A. Crampton, Z. Huang, J. Booth, Simulations of dissolution of spherical particles in laminar shear flow, Chemical Engineering Research and Design 93 (July) (2015) 66-78. doi:10.1016/j.cherd.2014.06.027.

URL http://dx.doi.org/10.1016/j.cherd.2014.06.027 http://linkinghub.elsevier.com/retrieve/pii/S0263876214003025

[9] D. Li, A. Wei, K. Luo, J. Fan, Direct numerical simulation of a particle-laden flow in a flat plate boundary layer, International Journal of Multiphase Flow 79 (2016) 124-143. doi:10.1016/j.ijmultiphaseflow.2015.10.011.

URL http://linkinghub.elsevier.com/retrieve/pii/S0301932215002505

[10] A. Soldati, C. Marchioli, Physics and modelling of turbulent particle deposition and entrainment: Review of a systematic study, International Journal of Multiphase Flow 35 (9) (2009) 827-839. doi:10.1016/j.ijmultiphaseflow.2009.02.016.

URL http://dx.doi.org/10.1016/j.ijmultiphaseflow.2009.02.016 http://linkinghub.elsevier.com/retrieve/pii/S030193220900032

[11] J. Capecelatro, O. Desjardins, An Euler-Lagrange strategy for simulating particle-laden flows, Journal of Computational Physics 238 (2013) 1-31. doi:10.1016/j.jcp.2012.12.015. URL http://dx.doi.org/10.1016/j.jcp.2012.12.015

[12] F. Municchi, S. Radl, Consistent closures for Euler-Lagrange models of bi-disperse gas-particle suspensions derived from particle-resolved direct numerical simulations, International Journal of Heat and Mass Transfer 111 (2017) 171-190. doi:10.1016/j.ijheatmasstransfer.2017.03.122. URL http://dx.doi.org/10.1016/j.ijheatmasstransfer.2017.03.122 http://linkinghub.elsevier.com/retrieve/pii/S0017931016338

[13] M.-S. Salehi, M. Askarishahi, S. Radl, Analytical solution for thermal transport in packed beds with volumetric heat source, Chemical Engineering Journal 316 (2017) 131-136. doi:10.1016/j.cej.2017.01.076 URL http://dx.doi.org/10.1016/j.cej.2017.01.076 http://linkinghub.elsevier.com/retrieve/pii/S1385894717300773

[14] S. Radl, S. Sundaresan, A drag model for filtered EulerLagrange simulations of clustered gasparticle suspensions, Chemical Engineering Science 117 (2014) 416-425. doi:10.1016/j.ces.2014.07.011.

[15] J. Theuerkauf, P. Witt, D. Schwesig, Analysis of particle porosity distribution in fixed beds using the discrete element method, Powder Technology 165 (2) (2006) 92-99. doi:10.1016/j.powtec.2006.03.022.

URL http://linkinghub.elsevier.com/retrieve/pii/S0032591006001069

[16] W. van Antwerpen, C. du Toit, P. Rousseau, A review of correlations to model the packing structure and effective thermal conductivity in packed beds of mono-sized spherical particles, Nuclear Engineering and Design 240 (7) (2010) 1803-1818. doi:10.1016/j.nucengdes.2010.03.009

URL http://dx.doi.org/10.1016/j.nucengdes.2010.03.009 http://linkinghub.elsevier.com/retrieve/pii/S002954931000155X

[17] V. M.H. Govindarao, G. F. Froment, Voidage profiles in packed beds of spheres, Chemical Engineering Science 41 (3) (1986) 533-539. doi:10.1016/0009-2509(86)87035-X.

[18] J. Kubie, Influence of containing walls on the distribution of voidage in packed beds of uniform spheres, Chemical Engineering Science 43 (6) (1988) 1403-1405. doi:10.1016/0009-2509(88)85113-3.

URL http://linkinghub.elsevier.com/retrieve/pii/0009250988851133

[19] A. de Klerk, Voidage variation in packed beds at small column to particle diameter ratio, AIChE Journal 49 (8) (2003) $2022-2029$. doi:10.1002/aic.690490812. URL http://doi.wiley.com/10.1002/aic.690490812

[20] G. E. Mueller, Radial void fraction distributions in randomly packed fixed beds of uniformly sized spheres in cylindrical containers, Powder Technology 77 (3) (1993) 313-319. doi:10.1016/0032-5910(93)85023-3. URL http://linkinghub.elsevier.com/retrieve/pii/0032591093850233

[21] A. Singhal, S. Cloete, S. Radl, R. Quinta-Ferreira, S. Amini, Heat transfer to a gas from densely packed beds of monodisperse spherical particles, Chemical Engineering Journal 314 (2017) 27-37. doi:10.1016/j.cej.2016.12.124.

URL http://dx.doi.org/10.1016/j.cej.2016.12.124 http://linkinghub.elsevier.com/retrieve/pii/S1385894716318976

[22] S. Bale, M. Sathe, O. Ayeni, A. S. Berrouk, J. Joshi, K. Nandakumar, Spatially resolved mass transfer coefficient for moder- 
ate Reynolds number flows in packed beds: Wall effects, International Journal of Heat and Mass Transfer 110 (2017) $406-415$. doi:10.1016/j.ijheatmasstransfer.2017.03.052.

URL http://linkinghub.elsevier.com/retrieve/pii/S0017931016339837

[23] M. Giese, K. Rottschäfer, D. Vortmeyer, Measured and modeled superficial flow profiles in packed beds with liquid flow, AIChE Journal 44 (2) (1998) 484-490. doi:10.1002/aic.690440225.

URL http://doi.wiley.com/10.1002/aic.690440225

[24] W. Holloway, X. Yin, S. Sundaresan, Fluid-particle drag in inertial polydisperse gas-solid suspensions, AIChE Journal 59 (2) (2009) 1995-2004. doi:10.1002/aic.12127.

URL http://doi.wiley.com/10.1002/aic.12127 http://www.ncbi.nlm.nih.gov/pubmed/23641116 http://www.pubmedcentral.nih.gov/articlerender.fcgi?artid=PMC3638963

[25] Y. Tang, E. A. J. F. Peters, J. A. M. Kuipers, Direct numerical simulations of dynamic gas-solid suspensions, AIChE Journal 62 (6) (2016) 1958-1969. arXiv:1402.6991v1, doi:10.1002/aic.15197. URL http://doi.wiley.com/10.1002/aic.15197

[26] S. Tenneti, B. Sun, R. Garg, S. Subramaniam, Role of fluid heating in dense gas-solid flow as revealed by particle-resolved direct numerical simulation, International Journal of Heat and Mass Transfer 58 (1-2) (2013) 471-479. doi:10.1016/j.ijheatmasstransfer.2012.11.006. URL http: //dx.doi .org/10.1016/j . ijheatmasstransfer.2012.11.006

[27] S. Whitaker, The Method of Volume Averaging, Vol. 13 of Theory and Applications of Transport in Porous Media, Springer Netherlands, Dordrecht, 1999. doi:10.1007/978-94-017-3389-2.

URL http://books.google.com/books?hl=fr\&lr=\&id=x7mQCEokSCAC\&pgis=1 http://link.springer.com/10.1007/978-94-017-3389-2

[28] U. Hornung, Homogenization and Porous Media, Vol. 6 of Interdisciplinary Applied Mathematics, Springer New York, New York, NY, 1997. doi:10.1007/978-1-4612-1920-0.

URL http: //link. springer. com/10.1007/978-1-4612-1920-0

[29] OpenFOAM, https://openfoam.org/. URL https: //openfoam.org/

[30] CFDEMproject, https://www.cfdem.com/. URL https: //www. cfdem. com/

[31] C. Kloss, C. Goniva, A. Hager, S. Amberger, S. Pirker, Models , algorithms and validation for opensource DEM and CFD-DEM, Progress in Computational Fluid Dynamics 12 (2012) 140-152. doi:10.1504/PCFD.2012.047457.

[32] B. Sun, S. Tenneti, S. Subramaniam, Modeling average gassolid heat transfer using particle-resolved direct numerical simulation, International Journal of Heat and Mass Transfer 86 (2015) 898-913. doi:10.1016/j.ijheatmasstransfer.2015.03.046. URL http://dx.doi.org/10.1016/j.ijheatmasstransfer.2015.03.046 http://linkinghub.elsevier.com/retrieve/pii/S0017931015003

[33] N. G. Deen, E. a. J. F. Peters, J. T. Padding, J. a. M. Kuipers, Review of direct numerical simulation of fluid-particle mass, momentum and heat transfer in dense gas-solid flows, Chemical Engineering Science 116 (2014) 710-724. doi:10.1016/j.ces.2014.05.039. URL http://dx.doi.org/10.1016/j.ces.2014.05.039

[34] S. H. L. Kriebitzsch, M. A. van der Hoef, J. A. M. Kuipers, Drag force in discrete particle models-Continuum scale or single particle scale?, AIChE Journal 59 (1) (2013) 316-324. doi:10.1002/aic.13804. URL http://doi.wiley.com/10.1002/aic.13804

[35] Z. Yu, X. Shao, A. Wachs, A fictitious domain method for particulate flows with heat transfer, Journal of Computational Physics 217 (2) (2006) 424-452. doi:10.1016/j.jcp.2006.01.016. URL http://linkinghub.elsevier.com/retrieve/pii/S0021999106000167

[36] H. Tavassoli, E. Peters, J. Kuipers, Direct numerical simulation of non-isothermal flow through dense bidisperse random arrays of spheres, Powder Technology 314 (2017) 291-298. doi:10.1016/j.powtec.2016.09.088. URL http://dx.doi.org/10.1016/j.powtec.2016.09.088 http://linkinghub.elsevier.com/retrieve/pii/S0032591016306684

[37] Z.-G. Feng, E. E. Michaelides, Heat transfer in particulate flows with Direct Numerical Simulation (DNS), International Journal of Heat and Mass Transfer 52 (3-4) (2009) 777-786. doi:10.1016/j.ijheatmasstransfer.2008.07.023.

URL http://linkinghub.elsevier.com/retrieve/pii/S0017931008004249

[38] F. Municchi, C. Goniva, S. Radl, Highly efficient spatial data filtering in parallel using the opensource library CPPPO, Computer Physics Communications 207 (2016) 400-414. doi:10.1016/j.cpc.2016.05.026. URL http://linkinghub.elsevier.com/retrieve/pii/S0010465516301606

[39] W. Zhang, K. E. Thompson, A. H. Reed, L. Beenken, Relationship between packing structure and porosity in fixed beds of equilateral cylindrical particles, Chemical Engineering Science 61 (24) (2006) 8060-8074. arXiv:/linkinghub.elsevier.com/retrieve/pii/S0960982205000989, doi:10.1016/j.ces.2006.09.036.

URL http://linkinghub.elsevier.com/retrieve/pii/S0009250906006166

[40] F. Municchi, S. Radl, C. Goniva, Near-wall effects for momentum, heat and mass transport in gas-particle suspensions at moderate Reynolds numbers, in: APS Division of Fluid Dynamics Meeting, 2016. doi:10.13140/RG.2.2.29649.74087.

URL https : //www.researchgate.net/publication/310607273_Near-wall_effects_for_momentum_heat_and_mass_transport_in_gas-partic 